\title{
Interpolation of compact bilinear operators among quasi-Banach spaces and applications
}

\author{
Fernando Cobos ${ }^{* 1}$, Luz M. Fernández-Cabrera ${ }^{* * 2}$, and Antón Martínez ${ }^{* * 3}$ \\ ${ }^{1}$ Departamento de Análisis Matemático y Matemática Aplicada, Facultad de Matemáticas, Universidad Com- \\ plutense de Madrid, Plaza de Ciencias 3, 28040 Madrid. Spain \\ ${ }^{2}$ Sección Departamental del Departamento de Análisis Matemático y Matemática Aplicada, Facultad de Estu- \\ dios Estadísticos, Universidad Complutense de Madrid, 28040 Madrid. Spain \\ ${ }^{3}$ Departamento de Matemática Aplicada I, Escuela de Ingeniería Industrial, Universidad de Vigo, 36200 Vigo, \\ Spain
}

Received ..., revised ..., accepted ...

Published online ...

Key words Compact bilinear operators, real interpolation of quasi-Banach couples, commutators of CalderónZygmund operators, interpolation of compact bilinear operators among $L_{p}$ spaces. MSC (2010) Primary 46M35, 47B07. Secondary 47B38, 42B20.

Dedicated to Professor Thomas Kühn on the occasion of his 65th birthday.

We study the interpolation properties of compact bilinear operators by the general real method among quasiBanach couples. As an application we show that commutators of Calderón-Zygmund bilinear operators $S$ : $L_{p} \times L_{q} \longrightarrow L_{r}$ are compact provided that $1 / 2<r<1,1<p, q<\infty$ and $1 / p+1 / q=1 / r$.

Copyright line will be provided by the publisher

\section{Introduction}

This paper refers to interpolation theory, a consolidated branch of functional analysis which has found important applications in harmonic analysis, partial differential equations and operator theory, as one can see in the monographs by Butzer and Berens [6], Bergh and Löfström [4], Triebel [44, 45], König [32] or Bennett and Sharpley [1]. Inside this theory, interpolation of compact linear operators is a very active research area. It started with the pioneering results of Krasnosel'skii [34], Lions and Peetre [37] and Persson [42] in the early 1960. Since then it has attracted the attention of many authors (see [9] and the references given there).

As for the real interpolation method $\left(A_{0}, A_{1}\right)_{\theta, q}$, it was a long standing problem to show that if any restriction of the operator is compact, then the interpolated operator is also compact. It was solved in 1992 by Cwikel [17] and Cobos, Kühn and Schonbek [13]. Later the result was extended to couples of quasi-Banach spaces by Cobos and Persson [15].

Interpolation properties of compact bilinear (or multilinear) operators were studied by Calderón [7] in his foundational paper on the complex interpolation method. The case of the real method has been investigated

\footnotetext{
* Corresponding author: e-mail: cobos@mat.ucm.es, Phone: +34913944453

** e-mail: luz_fernandez-c@mat.ucm.es, Phone: +34913944018

*** e-mail: antonmar@uvigo.es, Phone: +34986812 153
} 
much more recently by Fernandez and Silva [22] and by Fernández-Cabrera and Martínez [23, 24] by using the techniques developed by Cobos and Peetre [14] and Cobos, Kühn and Schonbek [13] to deal with linear operators.

Results of $[23,24]$ refer to the general real method which is defined by replacing the weighted $L_{q}$ norm used in $\left(A_{0}, A_{1}\right)_{\theta, q}$ by a more general lattice norm. The outcome is a very flexible method. For example, working with the couple $\left(L_{1}, L_{\infty}\right)$, the real method only produce Lebesgue spaces and Lorentz spaces. However, the general real method can generate any interpolation space with respect to $\left(L_{1}, L_{\infty}\right)$ (see [5] or [39]). In particular, Orlicz spaces and Lorentz-Zygmund spaces arise by using the general real method.

An important motivation for the investigations on interpolation properties of compact bilinear operators has been the fact that this kind of operators occurs rather naturally in harmonic analysis. This has been shown in the last few years in the papers by Bényi and Torres [3], Bényi and Oh [2], Hu [27] and other authors. In particular, Bényi and Torres [3] have established compactness of commutators $\mathbf{S}$ of Calderón-Zygmund bilinear operators (see Section 6 below) when acting from $L_{p} \times L_{q}$ into $L_{r}$ provided that $1<p, q<\infty, 1 \leq r<\infty$ and $1 / p+1 / q=1 / r$. These operators are bounded in a more broad range for the parameter $r$. Namely, for $1 / 2<r<\infty$ and $1 / p+1 / q=1 / r$ (see the papers by Lerner et al [36] and Pérez et al [41]). So, it is natural to wonder for compactness of $S$ in the range $1 / 2<r<1$, where the target space is no longer a Banach space but a quasi-Banach space and therefore duality arguments cannot be used. In this paper we solve this problem by means of interpolation techniques.

We start by reviewing in Section 2 the construction of the general real method for quasi-Banach couples. We also establish there some auxiliary results for our later considerations. In Section 3 we show the interpolation theorem for bounded bilinear operators, with a handy estimate for the norm of the interpolated operator. Then we review the properties of compact bilinear operators among quasi-Banach spaces and we prove other two auxiliary results. Section 4 contains the abstract results on interpolation of compact bilinear operators in the setting of the quasi-Banach spaces. The results extend those of Fernández-Cabrera and Martínez [23, 24] for the Banach case. We omit details when the arguments of $[23,24]$ need only minor modifications, but sometimes we must give separate proofs. Applications of these abstract results are given in the last two sections. In Section 5 we establish a reinforced version of an interpolation result of Calderón and Zygmund [8] on bounded bilinear operators among $L_{p}$ spaces. Finally, in Section 6, we prove compactness of commutators of bilinear Calderón-Zygmund operators $S: L_{p} \times L_{q} \longrightarrow L_{r}$ for $1<p, q<\infty, 1 / 2<r<1$ and $1 / p+1 / q=1 / r$.

\section{Real interpolation of quasi-Banach spaces}

Important spaces as the Lebesgue spaces $L_{p}$ or the Schatten-von Neumann operator spaces $S_{p}(H)$ are defined for $0<p<1$. Then they are not Banach spaces but quasi-Banach spaces, that is to say, the triangle inequality needs an additional constant $c \geq 1$.

Let $\left(A,\|\cdot\|_{A}\right)$ be a quasi-Banach space with constant $c=c_{A} \geq 1$ in the quasi-triangle inequality and let $0<p \leq 1$ such that $c=2^{1 / p-1}$. Then there is another quasi-norm $\||\cdot \||$ on $A$ which is equivalent to $\| \cdot \|_{A}$ and such that $\left\||\cdot \||^{p}\right.$ satisfies the triangle inequality (that is to say, $\||\cdot \||$ is a $p$-norm). See $[33, \S 5.10]$ or $[32$, Proposition 1.c.5]. We say that $A$ is a $p$-normed quasi-Banach space. Clearly, if $0<r<p$, then $A$ is also an $r$-normed quasi-Banach space.

By a (p-normed) quasi-Banach couple $\bar{A}=\left(A_{0}, A_{1}\right)$ we mean two ( $p$-normed) quasi-Banach spaces $A_{j}$ which are continuously embedded in the same Hausdorff topological vector space. Given $t>0$, Peetre's $K$ - and $J$-functionals are defined by

$$
K(t, a)=K\left(t, a ; A_{0}, A_{1}\right)=\inf \left\{\left\|a_{0}\right\|_{A_{0}}+t\left\|a_{1}\right\|_{A_{1}}: a=a_{0}+a_{1}, a_{j} \in A_{j}\right\}
$$


where $a \in A_{0}+A_{1}=\Sigma(\bar{A})$, and

$$
J(t, a)=J\left(t, a ; A_{0}, A_{1}\right)=\max \left\{\|a\|_{A_{0}}, t\|a\|_{A_{1}}\right\}, \quad a \in A_{0} \cap A_{1}=\Delta(\bar{A})
$$

(see $[4,44,5])$. Functionals $K(t, \cdot)$ and $J(t, \cdot)$ are quasi-norms in $\Sigma(\bar{A})$ and $\Delta(\bar{A})$, respectively. Note that we can take the same constant $c \geq 1$ in the quasi-triangle inequality for any $t>0$. The functional $K(1, \cdot)$ coincides with the quasi-norm $\|\cdot\|_{\Sigma(\bar{A})}$ of $\Sigma(\bar{A})$ and $J(1, \cdot)$ is $\|\cdot\|_{\Delta(\bar{A})}$.

Observe that if $\|\cdot\|_{A_{0}}$ and $\|\cdot\|_{A_{1}}$ are $p$-norms then $J(t, \cdot)$ is a $p$-norm on $\Delta(\bar{A})$, and the functional

$$
K_{p}(t, a)=\inf \left\{\left(\left\|a_{0}\right\|_{A_{0}}^{p}+t^{p}\left\|a_{1}\right\|_{A_{1}}^{p}\right)^{1 / p}: a=a_{0}+a_{1}, a_{j} \in A_{j}\right\}
$$

is a $p$-norm on $\Sigma(\bar{A})$, which is equivalent to $K(t, \cdot)$. Namely,

$$
K(t, a) \leq K_{p}(t, a) \leq 2^{1 / p} K(t, a), \quad a \in \Sigma(\bar{A}) .
$$

The general real interpolation method has been studied in the monographs by Peetre [40] and by Brudnyı̌ and Krugljak [5], and the articles by Cwikel and Peetre [18], Nilsson [38, 39], Cobos, Fernández-Cabrera, Manzano and Martínez [10] and Cobos, Fernández-Cabrera and Martínez [11, 12] among other papers. Following [38], here we consider this method realized in discrete way. Subsequently, by a quasi-Banach sequence lattice $\Gamma$ we mean a quasi-Banach space of real valued sequences with $\mathbb{Z}$ as index set which satisfies the following properties:

(i) $\Gamma$ contains all sequences with only finitely many non-zero co-ordinates.

(ii) Whenever $\left|\xi_{m}\right| \leq\left|\eta_{m}\right|$ for each $m \in \mathbb{Z}$ and $\left(\eta_{m}\right) \in \Gamma$, then $\left(\xi_{m}\right) \in \Gamma$ and $\left\|\left(\xi_{m}\right)\right\|_{\Gamma} \leq\left\|\left(\eta_{m}\right)\right\|_{\Gamma}$.

We say that $\Gamma$ is $K$-non-trivial if $\left(\min \left(1,2^{m}\right)\right) \in \Gamma$.

If $\bar{A}=\left(A_{0}, A_{1}\right)$ is a quasi-Banach couple and $\Gamma$ is $K$-non-trivial, the $K$-space $\bar{A}_{\Gamma ; K}=\left(A_{0}, A_{1}\right)_{\Gamma ; K}$ is formed of all $a \in \Sigma(\bar{A})$ such that $\left(K\left(2^{m}, a\right)\right) \in \Gamma$. We put

$$
\|a\|_{\bar{A}_{\Gamma ; K}}=\left\|\left(K\left(2^{m}, a\right)\right)\right\|_{\Gamma} .
$$

Since

$$
K\left(2^{m}, a\right) \leq \min \left(1,2^{m}\right) J(1, a), a \in \Delta(\bar{A}), m \in \mathbb{Z},
$$

and

$$
\min \left(1,2^{m}\right) K(1, a) \leq K\left(2^{m}, a\right), a \in \Sigma(\bar{A}), m \in \mathbb{Z}
$$

one can check that $\bar{A}_{\Gamma ; K}$ is an intermediate space with respect to $\bar{A}$, that is to say,

$$
A_{0} \cap A_{1} \hookrightarrow\left(A_{0}, A_{1}\right)_{\Gamma ; K} \hookrightarrow A_{0}+A_{1}
$$

Here $\hookrightarrow$ means continuous embeddings.

Let $\bar{A}=\left(A_{0}, A_{1}\right), \bar{B}=\left(B_{0}, B_{1}\right)$ be quasi-Banach couples. By $T \in \mathcal{L}(\bar{A}, \bar{B})$ we mean that $T$ is a linear operator from $\Sigma(\bar{A})$ into $\Sigma(\bar{B})$ whose restriction to each $A_{j}$ defines a bounded operator from $A_{j}$ into $B_{j}$ with quasi-norm $\|T\|_{A_{j}, B_{j}}, j=0,1$.

If $T \in \mathcal{L}(\bar{A}, \bar{B})$, it is not hard to check that the restriction

$$
T:\left(A_{0}, A_{1}\right)_{\Gamma ; K} \longrightarrow\left(B_{0}, B_{1}\right)_{\Gamma ; K}
$$


is bounded with quasi-norm

$$
\|T\|_{\left(A_{0}, A_{1}\right)_{\Gamma ; K},\left(B_{0}, B_{1}\right)_{\Gamma ; K}} \leq \max \left\{\|T\|_{A_{0}, B_{0}},\|T\|_{A_{1}, B_{1}}\right\} .
$$

A better estimate can be obtained if we know the behaviour of the norms of the shift operators on $\Gamma$ (see [11, 12]). Given $k \in \mathbb{Z}$, the shift operator $\tau_{k}$ is defined by $\tau_{k} \xi=\left(\xi_{m+k}\right)_{m \in \mathbb{Z}}$ for $\xi=\left(\xi_{m}\right)_{m \in \mathbb{Z}}$. In view of [11, Lemma 2.6], it will be useful for our aims to assume in what follows that $\tau_{k}$ is bounded in $\Gamma$ for all $k \in \mathbb{Z}$ and

$$
\lim _{n \rightarrow \infty} 2^{-n}\left\|\tau_{n}\right\|_{\Gamma, \Gamma}=0 \quad \text { and } \quad \lim _{n \rightarrow \infty}\left\|\tau_{-n}\right\|_{\Gamma, \Gamma}=0 .
$$

Following [12] we put

$$
f(t)=f_{\Gamma}(t)=\left\|\tau_{\left[\log _{2} t\right]}\right\|_{\Gamma, \Gamma}, \quad t>0,
$$

where the logarithm is taken in base 2 and $[\cdot]$ is the greatest integer function.

It follows from (2.1) that

$$
f(t)=o(\max (1, t)) .
$$

Let $M_{1}=\max \left(1,\left\|\tau_{1}\right\|_{\Gamma, \Gamma}\right), M_{2}=\sup \{f(t): 0<t \leq 1\}=\sup \left\{\left\|\tau_{-n}\right\|_{\Gamma, \Gamma}: n \geq 0\right\}$ and $M_{3}=\sup \{f(t) / t$ : $1 \leq t<\infty\}=\sup \left\{2^{-n}\left\|\tau_{n}\right\|_{\Gamma, \Gamma}: n \geq 0\right\}$. Using that $\left\|\tau_{m+k}\right\|_{\Gamma, \Gamma} \leq\left\|\tau_{m}\right\|_{\Gamma, \Gamma}\left\|\tau_{k}\right\|_{\Gamma, \Gamma}, m, k \in \mathbb{Z}$, one can easily derive that:

For any $s, t>0$, we have $f(s t) \leq M_{1} f(s) f(t)$. Hence, if

$$
s<t \text { we get that } f(s) \leq M_{1} M_{2} f(t) \text { and } f(t) / t \leq M_{1} M_{3} f(s) / s .
$$

The argument used in [12, Lemma 4.3] in the Banach case also work in the more general quasi-Banach case considered here with the effect that if $T \in \mathcal{L}(\bar{A}, \bar{B})$ then

$$
\|T\|_{\bar{A}_{\Gamma ; K}, \bar{B}_{\Gamma ; K}} \leq\left\{\begin{array}{l}
0 \text { if }\|T\|_{A_{j}, B_{j}}=0 \text { for } j=0 \text { or } 1, \\
f(2)\|T\|_{A_{0}, B_{0}} f\left(\|T\|_{A_{1}, B_{1}} /\|T\|_{A_{0}, B_{0}}\right) \text { otherwise. }
\end{array}\right.
$$

The following result is proved in [23, (5.2)] for Banach couples but the argument uses the Hahn-Banach theorem, so we give a new proof which is valid in the quasi-Banach case.

Subsequently, we write $\delta_{m}^{k}$ for the Kronecker delta. We also put $e_{0}=\left(\delta_{m}^{0}\right)_{m \in \mathbb{Z}}$.

Lemma 2.1 Let $\bar{A}=\left(A_{0}, A_{1}\right)$ be a quasi-Banach couple and let $\Gamma$ be a $K$-non-trivial quasi-Banach sequence lattice satisfying (2.1). Then there is a constant $C>0$ such that

$$
\sup _{0<t<\infty} \frac{K(t, a)}{f(t)} \leq C\|a\|_{\bar{A}_{\Gamma ; K}} \quad, \quad a \in \bar{A}_{\Gamma ; K} .
$$

Pro of. Given any $t>0$, we can choose $k \in \mathbb{Z}$ such that $2^{k} \leq t<2^{k+1}$. We have

$$
\begin{aligned}
K(t, a) & \leq 2 K\left(2^{k}, a\right)=\frac{2}{\left\|e_{0}\right\|_{\Gamma}}\left\|K\left(2^{k}, a\right) e_{0}\right\|_{\Gamma} \\
& \leq \frac{2}{\left\|e_{0}\right\|_{\Gamma}}\left\|\tau_{k}\right\|_{\Gamma, \Gamma}\left\|\tau_{-k}\left(K\left(2^{k}, a\right) e_{0}\right)\right\|_{\Gamma} \\
& \leq \frac{2}{\left\|e_{0}\right\|_{\Gamma}}\left\|\tau_{k}\right\|_{\Gamma, \Gamma}\left\|\left(K\left(2^{m}, a\right)\right)\right\|_{\Gamma} \\
& \leq \frac{2}{\left\|e_{0}\right\|_{\Gamma}} f(t)\|a\|_{\bar{A}_{\Gamma ; K}} .
\end{aligned}
$$


For $0<p \leq 1$, the quasi-Banach sequence lattice $\Gamma$ is said to be $(p, J)$-non-trivial if

$$
\sup \left\{\left(\sum_{m=-\infty}^{\infty}\left(\min \left(1,2^{-m}\right)\left|\xi_{m}\right|\right)^{p}\right)^{1 / p}:\left\|\left(\xi_{m}\right)\right\|_{\Gamma} \leq 1\right\}<\infty .
$$

Clearly, if $\Gamma$ is $(p, J)$-non-trivial then $\Gamma$ is also $(r, J)$-non-trivial for any $p \leq r \leq 1$.

If $\bar{A}=\left(A_{0}, A_{1}\right)$ is a $p$-normed quasi-Banach couple and $\Gamma$ is $(p, J)$-non-trivial, the $J$-space $\bar{A}_{\Gamma ; J}=$ $\left(A_{0}, A_{1}\right)_{\Gamma ; J}$ consists of all sums $\sum_{m=-\infty}^{\infty} u_{m}$ (convergence in $\Sigma(\bar{A})$ ), where $\left(u_{m}\right) \subseteq A_{0} \cap A_{1}$ and $\left(J\left(2^{m}, u_{m}\right)\right) \in$ $\Gamma$. The quasi-norm on $\bar{A}_{\Gamma ; J}$ is given by

$$
\|a\|_{\bar{A}_{\Gamma ; J}}=\inf \left\{\left\|\left(J\left(2^{m}, u_{m}\right)\right)\right\|_{\Gamma}: a=\sum_{m=-\infty}^{\infty} u_{m}\right\}
$$

Since $\Sigma(\bar{A})$ is a $p$-normed quasi-Banach space, if $\left(J\left(2^{m}, u_{m}\right)\right) \in \Gamma$ then the series $\sum_{m=-\infty}^{\infty} u_{m}$ is convergent in $\Sigma(\bar{A})$ because

$$
\sum_{m=-\infty}^{\infty}\left\|u_{m}\right\|_{\Sigma(\bar{A})}^{p} \leq \sum_{m=-\infty}^{\infty} \min \left(1,2^{-m}\right)^{p} J\left(2^{m}, u_{m}\right)^{p}<\infty
$$

The following estimate is useful.

Lemma 2.2 Let $\bar{A}=\left(A_{0}, A_{1}\right)$ be a p-normed quasi-Banach couple and let $\Gamma$ be a $(p, J)$-non-trivial quasiBanach sequence lattice satisfying (2.1). Then there is a constant $C>0$ such that

$$
\|a\|_{\bar{A}_{\Gamma ; J}} \leq C \inf _{t>0} f(t) J\left(t^{-1}, a\right) \quad, \quad a \in \Delta(\bar{A})
$$

Proof. Given $t>0$, take $k \in \mathbb{Z}$ such that $2^{-k} \leq t<2^{-k+1}$. If $a \in \Delta(\bar{A})$, using the representation $a=\sum_{m=-\infty}^{\infty} \delta_{m}^{k} a$ we get

$$
\begin{aligned}
\|a\|_{\bar{A}_{\Gamma ; J}} & \leq\left\|\left(J\left(2^{m}, \delta_{m}^{k} a\right)\right)\right\|_{\Gamma}=J\left(2^{k}, a\right)\left\|\left(\delta_{m}^{k}\right)\right\|_{\Gamma} \\
& =J\left(2^{k}, a\right)\left\|\tau_{-k} e_{0}\right\|_{\Gamma} \leq 2 J\left(t^{-1}, a\right)\left\|\tau_{-k}\right\|_{\Gamma, \Gamma}\left\|e_{0}\right\|_{\Gamma} \\
& =2 J\left(t^{-1}, a\right) f(t)\left\|e_{0}\right\|_{\Gamma} .
\end{aligned}
$$

Corollary 2.3 Let $\bar{A}=\left(A_{0}, A_{1}\right)$ be a p-normed quasi-Banach couple and let $\Gamma$ be a $(p, J)$-non-trivial quasiBanach sequence lattice satisfying (2.1). Then there is a constant $C>0$ such that

$$
\|a\|_{\bar{A}_{\Gamma ; J}} \leq C\|a\|_{A_{0}} f_{\Gamma}\left(\|a\|_{A_{1}} /\|a\|_{A_{0}}\right) \quad, \quad a \in \Delta(\bar{A})
$$

Proof. Take $t=\|a\|_{A_{1}} /\|a\|_{A_{0}}$ in Lemma 2.2.

It turns out that $\left(A_{0}, A_{1}\right)_{\Gamma ; K} \hookrightarrow\left(A_{0}, A_{1}\right)_{\Gamma ; J}$. The converse embedding depends on the boundedness of the Calderón transform

$$
\Lambda_{p}\left(\xi_{m}\right)=\left(\left(\sum_{k=-\infty}^{\infty}\left(\min \left(1,2^{m-k}\right)\left|\xi_{k}\right|\right)^{p}\right)^{1 / p}\right)_{m \in \mathbb{Z}} .
$$

Namely, if $\Lambda_{p}$ is bounded in $\Gamma$ then $\left(A_{0}, A_{1}\right)_{\Gamma ; J} \hookrightarrow\left(A_{0}, A_{1}\right)_{\Gamma ; K}$ (see [38, Lemma 2.5]). 
Sometimes in our later computations it is useful that $\bar{A}_{\Gamma ; K}=\bar{A}_{\Gamma ; J}$ with equivalence of quasi-norms. To get it, working with couples of p-normed spaces, we shall assume that

$\Gamma$ is $K$-non-trivial , $(p, J)$-non-trivial and the operator $\Lambda_{p}$ is bounded in $\Gamma$.

In that case we write $\bar{A}_{\Gamma}$ for any of the spaces $\bar{A}_{\Gamma ; K}$ or $\bar{A}_{\Gamma ; J}$ and we denote by $\|\cdot\|_{\bar{A}_{\Gamma}}$ any of the two quasi-norms. This however will not cause any confusion.

For $0<q \leq \infty$ we let $\ell_{q}$ be the usual space of $q$-summable scalar sequences with $\mathbb{Z}$ as index set. Let $\left(\lambda_{m}\right)$ be a sequence of positive numbers and let $\left(W_{m}\right)$ be a sequence of quasi-Banach spaces with the same constant $c \geq 1$ in the quasi-triangle inequality for any $W_{m}$. We put

$$
\ell_{q}\left(\lambda_{m} W_{m}\right)=\left\{w=\left(w_{m}\right): w_{m} \in W_{m} \quad \text { and } \quad\left(\lambda_{m}\left\|w_{m}\right\|_{W_{m}}\right) \in \ell_{q}\right\} .
$$

The quasi-norm in $\ell_{q}\left(\lambda_{m} W_{m}\right)$ is given by $\|w\|_{\ell_{q}\left(\lambda_{m} W_{m}\right)}=\left\|\left(\lambda_{m}\left\|w_{m}\right\|_{W_{m}}\right)\right\|_{\ell_{q}}$. Note that in $\ell_{q}\left(\lambda_{m} W_{m}\right)$ the quasi-triangle inequality holds with constant $2^{1 / q} c$. We define the space $\Gamma\left(\lambda_{m} W_{m}\right)$ similarly. If $W_{m}$ is equal to the scalar field $\mathbb{K}\left(\mathbb{K}=\mathbb{R}\right.$ or $\mathbb{C}$ ), then we simply write $\ell_{q}\left(\lambda_{m}\right)$.

Lemma 2.4 Let $0<q_{0}, q_{1} \leq \infty$ and let $\left(W_{m}\right)$ be a sequence of quasi-Banach spaces with the same constant in the quasi-triangle inequality, so $\left(l_{q_{0}}\left(W_{m}\right), l_{q_{1}}\left(2^{-m} W_{m}\right)\right)$ is a p-normed quasi-Banach couple for some $0<$ $p \leq 1$. If $\Gamma$ is a quasi-Banach sequence lattice satisfying (2.1) and (2.6), then we have with equivalence of quasi-norms

$$
\left(l_{q_{0}}\left(W_{m}\right), l_{q_{1}}\left(2^{-m} W_{m}\right)\right)_{\Gamma}=\Gamma\left(W_{m}\right) .
$$

Proof. Since $p \leq \min \left(q_{0}, q_{1}\right)$, we have that

$$
\left(l_{p}\left(W_{m}\right), l_{p}\left(2^{-m} W_{m}\right)\right)_{\Gamma} \hookrightarrow\left(l_{q_{0}}\left(W_{m}\right), l_{q_{1}}\left(2^{-m} W_{m}\right)\right)_{\Gamma} \hookrightarrow\left(l_{\infty}\left(W_{m}\right), l_{\infty}\left(2^{-m} W_{m}\right)\right)_{\Gamma} .
$$

Hence, it suffices to show that

$$
\Gamma\left(W_{m}\right) \hookrightarrow\left(l_{p}\left(W_{m}\right), l_{p}\left(2^{-m} W_{m}\right)\right)_{\Gamma} \quad \text { and } \quad\left(l_{\infty}\left(W_{m}\right), l_{\infty}\left(2^{-m} W_{m}\right)\right)_{\Gamma} \hookrightarrow \Gamma\left(W_{m}\right) .
$$

Let $w=\left(w_{m}\right) \in \Gamma\left(W_{m}\right)$ and write $u_{k}=\left(\delta_{m}^{k} w_{k}\right)_{m \in \mathbb{Z}}$ for the vector valued sequence having all co-ordinates equal to 0 except for the $k$-th one which is $w_{k}$. We have that $w=\sum_{k=-\infty}^{\infty} u_{k}$ and

$$
J\left(2^{k}, u_{k}\right)=\max \left(\left\|u_{k}\right\|_{\ell_{p}\left(W_{m}\right)}, 2^{k}\left\|u_{k}\right\|_{\ell_{p}\left(2-m W_{m}\right)}\right)=\left\|w_{k}\right\|_{W_{k}} .
$$

Hence

$$
\|w\|_{\left(\ell_{p}\left(W_{m}\right), \ell_{p}\left(2^{-m} W_{m}\right)\right)_{\Gamma}} \leq\left\|\left(J\left(2^{m}, u_{m}\right)\right)\right\|_{\Gamma}=\left\|\left(\left\|w_{m}\right\|_{W_{m}}\right)\right\|_{\Gamma}=\|w\|_{\Gamma\left(W_{m}\right)} .
$$

To establish the other embedding in (2.7) let

$$
w=\left(w_{m}\right) \in\left(\ell_{\infty}\left(W_{m}\right), \ell_{\infty}\left(2^{-m} W_{m}\right)\right)_{\Gamma}
$$

and take any representation $w=u+v$ where $u=\left(u_{m}\right) \in \ell_{\infty}\left(W_{m}\right)$ and $v=\left(v_{m}\right) \in \ell_{\infty}\left(2^{-m} W_{m}\right)$. Then

$$
\left\|w_{k}\right\|_{W_{k}} \leq c\left(\left\|u_{k}\right\|_{W_{k}}+\left\|v_{k}\right\|_{W_{k}}\right) \leq c\left(\|u\|_{\ell_{\infty}\left(W_{m}\right)}+2^{k}\|v\|_{\ell_{\infty}\left(2^{-m} W_{m}\right)}\right) .
$$

This implies that $\left\|w_{k}\right\|_{W_{k}} \leq c K\left(2^{k}, w\right)$ and therefore

$$
\|w\|_{\Gamma\left(W_{m}\right)}=\left\|\left(\left\|w_{m}\right\|_{W_{m}}\right)\right\|_{\Gamma} \leq c\left\|\left(K\left(2^{m}, w\right)\right)\right\|_{\Gamma}=c\|w\|_{\left(\ell_{\infty}\left(W_{m}\right), \ell_{\infty}\left(2^{-m} W_{m}\right)\right)_{\Gamma}} .
$$

This completes the proof. 
We end this section with some examples. Before them, we recall that two functions $f, g:(0, \infty) \longrightarrow(0, \infty)$ are said to be equivalent $(f \sim g)$ if there are positive constants $c_{1}, c_{2}$ such that $c_{1} g(t) \leq f(t) \leq c_{2} g(t)$ for all $t>0$. A function $\rho:(0, \infty) \longrightarrow(0, \infty)$ is said to be a function parameter if $\rho(t)$ increases from 0 to $\infty, \rho(t) / t$ decreases from $\infty$ to 0 and, for every $t>0, s_{\rho}(t)=\sup \{\rho(t s) / \rho(s): s>0\}$ is finite with $s_{\rho}(t)=o(\max (1, t))$ as $t \rightarrow 0$ and $t \rightarrow \infty$ (see $[26,28,43]$ ).

Example 2.5 Let $0<q \leq \infty$ and let $\rho$ be a function parameter. Then $\Gamma=\ell_{q}\left(1 / \rho\left(2^{m}\right)\right)$ is a quasiBanach sequence lattice. Shift operators in $\ell_{q}\left(1 / \rho\left(2^{m}\right)\right)$ satisfy $\left\|\tau_{k}\right\|_{\ell_{q}\left(1 / \rho\left(2^{m}\right)\right), \ell_{q}\left(1 / \rho\left(2^{m}\right)\right)} \leq s_{\rho}\left(2^{k}\right)$, so (2.1) is satisfied. Moreover, if $0<p \leq q$, the quasi-norm of the Calderón transform $\Lambda_{p}$ in $\ell_{q}\left(1 / \rho\left(2^{m}\right)\right)$ is bounded by the series $\left(\sum_{r=-\infty}^{\infty}\left(\min \left(1,2^{r}\right) s_{\rho}\left(2^{-r}\right)\right)^{p}\right)^{1 / p}$ which converges because for some $\delta>0$ we have $s_{\rho}(t)=$ $O\left(\max \left(t^{\delta}, t^{1-\delta}\right)\right)$ (see [43, Proposition 1.3]). The space $\ell_{q}\left(1 / \rho\left(2^{m}\right)\right)$ is also $(p, J)$-non-trivial and $K$-nontrivial. The interpolation method generated by $\ell_{q}\left(1 / \rho\left(2^{m}\right)\right)$ is known in the literature as the real method with a function parameter $\left(A_{0}, A_{1}\right)_{\ell_{q}\left(1 / \rho\left(2^{m}\right)\right)}=\left(A_{0}, A_{1}\right)_{\rho, q}$. It has been studied in [26, 28, 43] among other papers.

Example 2.6 Let $g:(0, \infty) \longrightarrow(0, \infty)$ be a measurable function which is equivalent to a function parameter $\rho$ and let $0<q \leq \infty$. Then $\Gamma=\ell_{q}\left(1 / g\left(2^{m}\right)\right)$ is also a quasi-Banach sequence lattice. If we choose $g(t)=$ $t^{\theta}(1+|\log t|)^{\mathbb{A}}$ where $0<\theta<1, \mathbb{A}=\left(\alpha_{0}, \alpha_{\infty}\right) \in \mathbb{R}^{2}$ and

$$
(1+|\log t|)^{\mathbb{A}}=\left\{\begin{array}{lll}
(1-\log t)^{-\alpha_{0}} & \text { if } & 0<t \leq 1 \\
(1+\log t)^{-\alpha_{\infty}} & \text { if } & 1<t<\infty
\end{array}\right.
$$

then we obtain logarithmic interpolation spaces, studied in $[20,21,19,16]$. Note that here it is not allow that $\theta$ takes the values 0 or 1 because we want that (2.1) and (2.6) are satisfied.

Example 2.7 Let $0<\theta<1$. The special case in Example 2.5 when $\rho(t)=t^{\theta}$ gives the classical real interpolation method $\left(A_{0}, A_{1}\right)_{\theta, q}($ see $[4,44,1,5])$.

\section{Bilinear operators}

Let $A, B, E$ be quasi-Banach spaces and let $T: A \times B \longrightarrow E$ be a bilinear operator. The operator $T$ is said to be bounded if

$$
\|T\|_{A \times B, E}=\sup \left\{\|T(a, b)\|_{E}:\|a\|_{A} \leq 1,\|b\|_{B} \leq 1\right\}<\infty .
$$

We write $\mathcal{B}(A \times B, E)$ for the set of all bounded bilinear operators from $A \times B$ into $E$.

Let $\bar{A}=\left(A_{0}, A_{1}\right), \bar{B}=\left(B_{0}, B_{1}\right), \bar{E}=\left(E_{0}, E_{1}\right)$ be quasi-Banach couples. We write $T: \bar{A} \times \bar{B} \longrightarrow \bar{E}$ to mean that $T$ is a bounded bilinear operator $T \in \mathcal{B}(\Sigma(\bar{A}) \times \Sigma(\bar{B}), \Sigma(\bar{E}))$ such that for $j=0,1$, the restriction of $T$ to $A_{j} \times B_{j}$ defines a bounded bilinear operator $T \in \mathcal{B}\left(A_{j} \times B_{j}, E_{j}\right)$.

Next we describe the interpolation properties of bounded bilinear operators by the general real method.

Given two sequences $\xi=\left(\xi_{m}\right)_{m \in \mathbb{Z}}, \eta=\left(\eta_{m}\right)_{m \in \mathbb{Z}}$ of non-negative scalars, we define their convolution by the sequence $\xi \star \eta=\left(\sum_{k=-\infty}^{\infty} \xi_{k} \eta_{m-k}\right)_{m \in \mathbb{Z}}$. If $0<r \leq 1$, we write $\xi^{r}=\left(\xi_{m}^{r}\right)_{m \in \mathbb{Z}}$.

Theorem 3.1 Let $\bar{A}=\left(A_{0}, A_{1}\right)$ be a quasi-Banach couple, let $\bar{B}=\left(B_{0}, B_{1}\right)$ be a p-normed quasi-Banach couple and let $\bar{E}=\left(E_{0}, E_{1}\right)$ be an r-normed quasi-Banach couple $(0<p, r \leq 1)$. Assume that $\Gamma_{0}$ and $\Gamma_{2}$ are $K$-non-trivial quasi-Banach sequence lattices and $\Gamma_{1}$ is a $(p, J)$-non-trivial quasi-Banach sequence lattice satisfying (2.1). Furthermore, we suppose that there is a constant $M>0$ such that for all non-negative scalar sequences $\xi \in \Gamma_{0}$ and $\eta \in \Gamma_{1}$ we have

$$
\left\|\left(\xi^{r} \star \eta^{r}\right)^{1 / r}\right\|_{\Gamma_{2}} \leq M\|\xi\|_{\Gamma_{0}}\|\eta\|_{\Gamma_{1}} .
$$


Let $T: \bar{A} \times \bar{B} \longrightarrow \bar{E}$ and put $\|T\|_{j}=\|T\|_{A_{j} \times B_{j}, E_{j}}, j=0,1$. Then the restriction of $T$ to $\bar{A}_{\Gamma_{0} ; K} \times \bar{B}_{\Gamma_{1} ; J}$ defines a bounded bilinear operator $T: \bar{A}_{\Gamma_{0} ; K} \times \bar{B}_{\Gamma_{1} ; J} \longrightarrow \bar{E}_{\Gamma_{2} ; K}$ with

$$
\|T\|_{\bar{A}_{\Gamma_{0} ; K} \times \bar{B}_{\Gamma_{1} ; J}, \bar{E}_{\Gamma_{2} ; K}} \leq \begin{cases}0 & \text { if } \quad\|T\|_{j}=0, j=0 \text { or } 1 \\ C\|T\|_{0} f_{\Gamma_{1}}\left(\|T\|_{1} /\|T\|_{0}\right) & \text { otherwise. }\end{cases}
$$

Here $C$ is a constant independent of $T$.

Proof. Let $\sigma_{j}>\|T\|_{j}, j=0,1$ and choose $n \in \mathbb{Z}$ such that $2^{n} \leq \sigma_{1} / \sigma_{0}<2^{n+1}$. Take any $a \in \bar{A}_{\Gamma_{0} ; K}$, any $u \in B_{0} \cap B_{1}$ and $m, k \in \mathbb{Z}$. If $a=a_{0}+a_{1}$ with $a_{j} \in A_{j}$, we get

$$
\begin{aligned}
K\left(2^{m}, T(a, u)\right) & \leq\left\|T\left(a_{0}, u\right)\right\|_{E_{0}}+2^{m}\left\|T\left(a_{1}, u\right)\right\|_{E_{1}} \\
& \leq \sigma_{0}\left\|a_{0}\right\|_{A_{0}}\|u\|_{B_{0}}+2^{m-k-n} 2^{k+n} \sigma_{1}\left\|a_{1}\right\|_{A_{1}}\|u\|_{B_{1}} \\
& \leq \max \left(\sigma_{0}, 2^{-n} \sigma_{1}\right)\left(\left\|a_{0}\right\|_{A_{0}}+2^{m-k}\left\|a_{1}\right\|_{A_{1}}\right) J\left(2^{k+n}, u\right) .
\end{aligned}
$$

Taking the infimum over all possible decompositions $a=a_{0}+a_{1}$ with $a_{j} \in A_{j}$ and having in mind the choice of $n$ we get

$$
K\left(2^{m}, T(a, u)\right) \leq 2 \sigma_{0} K\left(2^{m-k}, a\right) J\left(2^{k+n}, u\right) .
$$

Take $b \in \bar{B}_{\Gamma_{1} ; J}$ and let $b=\sum_{k=-\infty}^{\infty} u_{k}$ any $J$-representation of $b$. Then in $\Sigma(\bar{B})$ we also have that $b=$ $\sum_{k=-\infty}^{\infty} u_{k+n}$. Moreover, since $K_{r}\left(t, \cdot ; E_{0}, E_{1}\right)$ is an $r$-norm on $\Sigma(\bar{E})$ which is equivalent to $K\left(t, . ; E_{0}, E_{1}\right)$, we obtain that $K\left(2^{m}, T(a, b)\right) \leq C_{1}\left(\sum_{k=-\infty}^{\infty} K\left(2^{m}, T\left(a, u_{k+n}\right)\right)^{r}\right)^{1 / r}$. Using (3.2) and (3.1), we derive that

$$
\begin{aligned}
\|T(a, b)\|_{\bar{E}_{\Gamma_{2} ; K}} & \leq C_{1}\left\|\left(\sum_{k=-\infty}^{\infty} K\left(2^{m}, T\left(a, u_{k+n}\right)\right)^{r}\right)^{1 / r}\right\|_{\Gamma_{2}} \\
& \leq 2 C_{1} \sigma_{0}\left\|\left(\sum_{k=-\infty}^{\infty} K\left(2^{m-k}, a\right)^{r} J\left(2^{k+n}, u_{k+n}\right)^{r}\right)^{1 / r}\right\|_{\Gamma_{2}} \\
& =2 C_{1} \sigma_{0}\left\|\left(\sum_{j=-\infty}^{\infty} K\left(2^{j}, a\right)^{r} J\left(2^{m+n-j}, u_{m+n-j}\right)^{r}\right)^{1 / r}\right\|_{\Gamma_{2}} \\
& \leq 2 C_{1} M \sigma_{0}\left\|\left(K\left(2^{m}, a\right)\right)\right\|_{\Gamma_{0}}\left\|\left(J\left(2^{m+n}, u_{m+n}\right)\right)\right\|_{\Gamma_{1}} \\
& \leq 2 C_{1} M \sigma_{0}\left\|\tau_{n}\right\|_{\Gamma_{1} \Gamma_{1}}\|a\|_{\bar{A}_{\Gamma_{0} ; K}}\left\|\left(J\left(2^{m}, u_{m}\right)\right)\right\|_{\Gamma_{1}} .
\end{aligned}
$$

Since $\left\|\tau_{n}\right\|_{\Gamma_{1}, \Gamma_{1}}=f_{\Gamma_{1}}\left(\sigma_{1} / \sigma_{0}\right)$, we get that

$$
\|T\|_{\bar{A}_{\Gamma_{0} ; K} \times \bar{B}_{\Gamma_{1} ; J}, \bar{E}_{\Gamma_{2} ; K}} \leq C \sigma_{0} f_{\Gamma_{1}}\left(\sigma_{1} / \sigma_{0}\right) .
$$

Now, if $\|T\|_{j}=0$ for $j=0$ or 1, letting $\sigma_{j} \rightarrow 0$ and using (2.2) we derive that $\|T\|_{\bar{A}_{\Gamma_{0} ; K} \times \bar{B}_{\Gamma_{1} ; J}, \bar{E}_{\Gamma_{2} ; K}}=0$. If $\|T\|_{j} \neq 0$ for $j=0,1$, taking $\sigma_{j}=(1+\varepsilon)\|T\|_{j}$ and letting $\varepsilon \rightarrow 0$ we conclude that $\|T\|_{\bar{A}_{\Gamma_{0} ; K} \times \bar{B}_{\Gamma_{1} ; J}, \bar{E}_{\Gamma_{2} ; K}} \leq$ $C\|T\|_{0} f_{\Gamma_{1}}\left(\|T\|_{1} /\|T\|_{0}\right)$.

Writing down Theorem 3.1 for $\bar{A}, \bar{B}, \bar{E}$ Banach couples, $p=r=1$ and $\Gamma_{0}, \Gamma_{1}, \Gamma_{2}$ Banach sequence lattices satisfying (2.1), (2.6) and (3.1), we recover [23, Theorem 3.1].

Theorem 3.1 applies to the real method with a function parameter (Example 2.5) and the real method (Example 2.7). Previous results on interpolation of bilinear operators by the real method among quasi-Banach couples can be found in the papers by Karadzhov [30] and König [31]. 
Let $A, B, E$ be quasi-Banach spaces. We say that $T \in \mathcal{B}(A \times B, E)$ is compact if for any bounded sets $V \subseteq A$ and $W \subseteq B$, we have that the closure of $T(V, W)=\{T(a, b): a \in V, b \in W\}$ is compact in $E$. We put $\mathcal{K}(A \times B, E)$ for the collection of all compact operators from $A \times B$ into $E$.

It is not hard to check that compactness of $T \in \mathcal{B}(A \times B, E)$ is equivalent to the fact that $T\left(U_{A}, U_{B}\right)$ is precompact in $E$. Here $U_{A}$ is the closed unit ball of $A$ and $U_{B}$ the corresponding ball of $B$. Moreover, as in the Banach case (see [3, Proposition 1]), $T \in \mathcal{B}(A \times B, E)$ is compact if, and only if, for any bounded sequences $\left(a_{n}\right) \subseteq A,\left(b_{n}\right) \subseteq B$, the sequence $\left(T\left(a_{n}, b_{n}\right)\right)$ has a convergent subsequence.

Using the characterization of compactness by sequences, it is not hard to check that if $T \in \mathcal{K}(A \times B, E)$, $E_{1}$ is another quasi-Banach space and $R$ is a bounded linear operator $R \in \mathcal{L}\left(E, E_{1}\right)$, then $R T=R \circ T \in$ $\mathcal{K}\left(A \times B, E_{1}\right)$. Moreover, if $A_{1}, B_{1}$ are quasi-Banach spaces and $R_{1}, R_{2}$ are bounded linear operators $R_{1} \in$ $\mathcal{L}\left(A_{1}, A\right), R_{2} \in \mathcal{L}\left(B_{1}, B\right)$, then $T \circ\left(R_{1}, R_{2}\right)(a, b)=T\left(R_{1}, R_{2}\right)(a, b)=T\left(R_{1} a, R_{2} b\right)$ belongs to $\mathcal{K}\left(A_{1} \times\right.$ $\left.B_{1}, E\right)$. It is also clear that if $T_{1}, T_{2} \in \mathcal{K}(A \times B, E)$ and $\alpha, \beta \in \mathbb{K}$, then $T=\alpha T_{1}+\beta T_{2} \in \mathcal{K}(A \times B, E)$.

Minor changes in the arguments given by Bényi and Torres [3, Proposition 3] for the Banach case, show that if $\left(T_{n}\right) \subseteq \mathcal{K}(A \times B, E)$ and $\left(T_{n}\right)$ converges to the bounded bilinear operator $T \in \mathcal{B}(A \times B, E)$ then $T \in \mathcal{K}(A \times B, E)$. In what follows, we will use freely all these properties of compact bilinear operators.

The following results will be useful in the proof of the main interpolation theorem of the next section. We write $c_{E}$ for the constant in the quasi-triangle inequality in the space $E$.

Lemma 3.2 Let $A, B, E, Z$ be quasi-Banach spaces, let $D$ be a dense subspace of $A$ and let $V$ be a dense subspace of $B$. Assume that $T \in \mathcal{K}(A \times B, E)$ is a compact bilinear operator and let $S_{n} \in \mathcal{L}(E, Z)$ be a bounded linear operator for each $n \in \mathbb{N}$ such that $\sup _{n \in \mathbb{N}}\left\|S_{n}\right\|_{E, Z}=M<\infty$. If $\lim _{n \rightarrow \infty}\left\|S_{n} T u\right\|_{Z}=0$ for all $u \in D \times V$, then $\lim _{n \rightarrow \infty}\left\|S_{n} T\right\|_{A \times B, Z}=0$.

Pro of. Using compactness of $T$ and density of $D$ in $A$ and of $V$ in $B$, given any $\varepsilon>0$, we can find a finite set $\left\{u_{1}, \ldots, u_{r}\right\} \subseteq D \times V$ with $u_{j}=\left(a_{j}, b_{j}\right),\left\|a_{j}\right\|_{A_{j}} \leq 1,\left\|b_{j}\right\|_{B_{j}} \leq 1$ and such that

$$
T\left(U_{A}, U_{B}\right) \subseteq \bigcup_{j=1}^{r}\left\{T u_{j}+\frac{\varepsilon}{2 M c_{Z}} U_{E}\right\} .
$$

By the assumption on $\left(S_{n}\right)$, there exists $N \in \mathbb{N}$ such that for any $n \geq N$ and any $1 \leq j \leq r$, we have that $\left\|S_{n} T u_{j}\right\|_{Z} \leq \varepsilon / 2 c_{Z}$. Consequently, given any $u \in U_{A} \times U_{B}$ if we choose $1 \leq j \leq r$ such that $\left\|T u-T u_{j}\right\|_{E} \leq$ $\varepsilon / 2 M c_{Z}$, then we obtain for $n \geq N$ that

$$
\left\|S_{n} T u\right\|_{Z} \leq c_{Z}\left(\left\|S_{n}\left(T u-T u_{j}\right)\right\|_{Z}+\left\|S_{n} T u_{j}\right\|_{Z}\right) \leq c_{Z} M\left\|T u-T u_{j}\right\|_{E}+\varepsilon / 2 \leq \varepsilon .
$$

Lemma 3.3 Let $\bar{A}=\left(A_{0}, A_{1}\right), \bar{B}=\left(B_{0}, B_{1}\right), \bar{E}=\left(E_{0}, E_{1}\right)$ be quasi-Banach couples and let $A, B, E$ be intermediate spaces with respect to $\bar{A}, \bar{B}, \bar{E}$, respectively. Assume that $T: \Sigma(\bar{A}) \times \Sigma(\bar{B}) \longrightarrow \Sigma(\bar{E})$ is bounded with $T \in \mathcal{K}(A \times B, E)$. Let $X, Y$ be quasi-Banach spaces and let $R_{n} \in \mathcal{L}(X, A), S_{n} \in \mathcal{L}(Y, B)$ such that $\sup _{n \in \mathbb{N}}\left\|R_{n}\right\|_{X, A}=M<\infty, \sup _{n \in \mathbb{N}}\left\|S_{n}\right\|_{Y, B}=L<\infty$ and $\lim _{n \rightarrow \infty}\left\|T\left(R_{n}, S_{n}\right)\right\|_{X \times Y, \Sigma(\bar{E})}=0$. Then

$$
\lim _{n \rightarrow \infty}\left\|T\left(R_{n}, S_{n}\right)\right\|_{X \times Y, E}=0 .
$$

Pro of. We proceed by contradiction. Since

$$
\sup _{n \in \mathbb{N}}\left\|T\left(R_{n}, S_{n}\right)\right\|_{X \times Y, E} \leq M L\|T\|_{A \times B, E}<\infty,
$$

Copyright line will be provided by the publisher 
if $\lim _{n \rightarrow \infty}\left\|T\left(R_{n}, S_{n}\right)\right\|_{X \times Y, E} \neq 0$ then we can find $\lambda>0$, a subsequence $\left(n^{\prime}\right)$ and vectors $\left(x_{n^{\prime}}\right) \subseteq U_{X},\left(y_{n^{\prime}}\right) \subseteq$ $U_{Y}$ such that

$$
\lim _{n^{\prime} \rightarrow \infty}\left\|T\left(R_{n^{\prime}} x_{n^{\prime}}, S_{n^{\prime}} y_{n^{\prime}}\right)\right\|_{E}=\lambda .
$$

The assumption on $\left(R_{n}\right)$ and $\left(S_{n}\right)$ yields that the sequence $\left(R_{n^{\prime}} x_{n^{\prime}}\right)$ is bounded in $A$ and $\left(S_{n^{\prime}} y_{n^{\prime}}\right)$ in $B$. Compactness of $T: A \times B \longrightarrow E$ implies, passing to another subsequence if necessary, that $\left(T\left(R_{n^{\prime \prime}} x_{n^{\prime \prime}}, S_{n^{\prime \prime}} y_{n^{\prime \prime}}\right)\right)$ converges to some $w$ in $E$. So $\|w\|_{E}=\lambda>0$ and $\left(T\left(R_{n^{\prime \prime}} x_{n^{\prime \prime}}, S_{n^{\prime \prime}} y_{n^{\prime \prime}}\right)\right)$ converges also to $w$ in $E_{0}+E_{1}$. However, using that $\lim _{n \rightarrow \infty}\left\|T\left(R_{n}, S_{n}\right)\right\|_{X \times Y, \Sigma(\bar{E})}=0$, we get that $\left(T\left(R_{n^{\prime \prime}} x_{n^{\prime \prime}}, S_{n^{\prime \prime}} y_{n^{\prime \prime}}\right)\right) \rightarrow 0$ in $E_{0}+E_{1}$, which contradicts that $w \neq 0$.

\section{Interpolation of compact bilinear operators}

Using Lemma 2.1 the arguments in [23, Theorem 5.1] can be modified to give the following.

Theorem 4.1 Let $\Gamma_{0}, \Gamma_{1}$ be K-non-trivial quasi-Banach sequence lattices satisfying (2.1). Let $\bar{A}=\left(A_{0}, A_{1}\right)$, $\bar{B}=\left(B_{0}, B_{1}\right)$ be quasi-Banach couples and let $E$ be a quasi-Banach space. Assume that $T \in \mathcal{B}(\Sigma(\bar{A}) \times$ $\Sigma(\bar{B}), E)$ with $T: A_{j} \times B_{j} \longrightarrow$ E compact for $j=0$ or 1 . Then $T: \bar{A}_{\Gamma_{0} ; K} \times \bar{B}_{\Gamma_{1} ; K} \longrightarrow E$ is also compact.

The next result can be derived by using (2.5) and proceeding as in [23, Theorem 5.3] for the Banach case.

Theorem 4.2 Let $\Gamma$ be a $(p, J)$-non-trivial quasi-Banach sequence lattice $(0<p \leq 1)$ satisfying (2.1). Assume that $A, B$ are quasi-Banach spaces and let $\bar{E}=\left(E_{0}, E_{1}\right)$ be a p-normed quasi-Banach couple. If $T \in \mathcal{B}(A \times B, \Delta(\bar{E}))$ satisfies that $T: A \times B \longrightarrow E_{j}$ is compact for $j=0$ or 1 , then $T: A \times B \longrightarrow \bar{E}_{\Gamma ; J}$ is compact as well.

Writing down these results for the real method with a function parameter (Example 2.5) we get the following.

Corollary 4.3 Let $\bar{A}=\left(A_{0}, A_{1}\right), \bar{B}=\left(B_{0}, B_{1}\right)$ be quasi-Banach couples and let $E$ be a quasi-Banach space. Assume that $T \in \mathcal{B}(\Sigma(\bar{A}) \times \Sigma(\bar{B}), E)$ satisfies that $T: A_{j} \times B_{j} \longrightarrow E$ is compact for $j=0$ or 1 . Then, for any $0<q_{0}, q_{1} \leq \infty$ and any function parameters $\rho_{0}, \rho_{1}$ we have that

$$
T:\left(A_{0}, A_{1}\right)_{\rho_{0}, q_{0}} \times\left(B_{0}, B_{1}\right)_{\rho_{1} ; q_{1}} \longrightarrow E
$$

is compact as well.

Corollary 4.4 Let $A, B$ are quasi-Banach spaces and let $\bar{E}=\left(E_{0}, E_{1}\right)$ be a quasi-Banach couple. Assume that $T \in \mathcal{B}(A \times B, \Delta(\bar{E}))$ satisfies that $T: A \times B \longrightarrow E_{j}$ is compact for $j=0$ or 1 . Then, for any $0<q \leq \infty$ and any function parameter $\rho$, we have that

$$
T: A \times B \longrightarrow\left(E_{0}, E_{1}\right)_{\rho, q}
$$

is also compact.

In particular, taking the function parameters as power functions, we derive the following interpolation result for the real method.

Corollary 4.5 Let $\bar{A}=\left(A_{0}, A_{1}\right), \bar{B}=\left(B_{0}, B_{1}\right)$ be quasi-Banach couples and let $E$ be a quasi-Banach space. Let $T \in \mathcal{B}(\Sigma(\bar{A}) \times \Sigma(\bar{B}), E)$ such that $T: A_{j} \times B_{j} \longrightarrow E$ is compact for $j=0$ or 1 . Then, for any $0<q_{0}, q_{1} \leq \infty$ and any $0<\theta_{0}, \theta_{1}<1$, we have that

$$
T:\left(A_{0}, A_{1}\right)_{\theta_{0}, q_{0}} \times\left(B_{0}, B_{1}\right)_{\theta_{1} ; q_{1}} \longrightarrow E
$$

is compact as well. 
Note that in Corollary 4.5 no relationship is assumed between parameters $\theta_{0}$ and $\theta_{1}$. Such freedom will be useful in the application given in Section 5.

Corollary 4.6 Let $A, B$ be quasi-Banach spaces and let $\bar{E}=\left(E_{0}, E_{1}\right)$ be a quasi-Banach couple. Assume that $T \in \mathcal{B}(A \times B, \Delta(\bar{E}))$ with $T: A \times B \longrightarrow E_{j}$ compact for $j=0$ or 1 . Then, for any $0<q \leq \infty$ and any $0<\theta<1$, we have that

$$
T: A \times B \longrightarrow\left(E_{0}, E_{1}\right)_{\theta, q}
$$

is also compact.

Now we prove the main result of this section.

Theorem 4.7 Let $\bar{A}=\left(A_{0}, A_{1}\right), \bar{B}=\left(B_{0}, B_{1}\right)$ be p-normed quasi-Banach couples $(0<p \leq 1)$, let $\bar{E}=\left(E_{0}, E_{1}\right)$ be an $r$-normed quasi-Banach couple $(0<r \leq 1)$ and let $\Gamma_{0}, \Gamma_{1}, \Gamma_{2}$ be quasi-Banach sequence lattices. We suppose that $\Gamma_{0}$ and $\Gamma_{1}$ satisfy (2.6) and that $\Gamma_{2}$ satisfies (2.1) and (2.6) with parameter $r$. Assume also that the sequence spaces satisfy the condition (3.1) on convolutions. Let $T: \bar{A} \times \bar{B} \longrightarrow \bar{E}$ such that any of the restrictions $T: A_{j} \times B_{j} \longrightarrow E_{j}$ is compact for $j=0$ or $j=1$. Then $T: \bar{A}_{\Gamma_{0}} \times \bar{B}_{\Gamma_{1}} \longrightarrow \bar{E}_{\Gamma_{2}}$ is also compact.

Proof. Let $A_{j}^{\circ}$ be the closure of $\Delta(\bar{A})$ in $A_{j}$. The couple $\overline{A^{\circ}}=\left(A_{0}^{\circ}, A_{1}^{\circ}\right)$ is also a $p$-normed quasi-Banach couple. Moreover, using the $J$-representation of $\bar{A}_{\Gamma_{0}}$, it is not difficult to check that $\left(A_{0}, A_{1}\right)_{\Gamma_{0}}=\left(A_{0}^{\circ}, A_{1}^{\circ}\right)_{\Gamma_{0}}=$ $\overline{A^{\circ}} \Gamma_{0}$. Similarly, $\left(B_{0}, B_{1}\right)_{\Gamma_{1}}=\left(B_{0}^{\circ}, B_{1}^{\circ}\right)_{\Gamma_{1}}=\overline{B^{\circ}} \Gamma_{1}$. Note also that the operator $T$ satisfies that $T: \overline{A^{\circ}} \times \overline{B^{\circ}} \longrightarrow$ $\bar{E}$, with $T: A_{j}^{\circ} \times B_{j}^{\circ} \longrightarrow E_{j}$ being compact provided that $T: A_{j} \times B_{j} \longrightarrow E_{j}$ is so. This allows us to work with the couples $\overline{A^{\circ}}, \overline{B^{\circ}}$ instead of $\bar{A}, \bar{B}$.

For $m \in \mathbb{Z}$, consider the $p$-normed spaces

$$
\begin{gathered}
F_{m}=\left(A_{0}^{\circ} \cap A_{1}^{\circ}, J\left(2^{m}, \cdot ; A_{0}^{\circ}, A_{1}^{\circ}\right)\right), \quad G_{m}=\left(B_{0}^{\circ} \cap B_{1}^{\circ}, J\left(2^{m}, \cdot ; B_{0}^{\circ}, B_{1}^{\circ}\right)\right) \\
\text { and } W_{m}=\left(E_{0}+E_{1}, K_{r}\left(2^{m}, \cdot ; E_{0}, E_{1}\right)\right) .
\end{gathered}
$$

These vector-valued sequence spaces are closely linked with the construction of the general real method. Namely, if we realized $\overline{A^{\circ}} \Gamma_{0}$ by means of the $J$-functional, then the map $\pi\left(u_{m}\right)=\sum_{m=-\infty}^{\infty} u_{m}$ (convergence in $\Sigma\left(\overline{A^{\circ}}\right)$ ) is surjective from $\Gamma_{0}\left(F_{m}\right)$ into $\overline{A^{\circ}} \Gamma_{0}$ and it induces the quasi-norm $\|\cdot\|_{\bar{A}^{\circ} \Gamma_{0} ; J}$. Moreover, since $A_{j}^{\circ}$ is $p$-normed, we also have that $\pi: \ell_{p}\left(2^{-j m} F_{m}\right) \longrightarrow A_{j}^{\circ}$ is bounded for $j=0,1$. Similarly, $\pi: \Gamma_{1}\left(G_{m}\right) \longrightarrow \bar{B}^{\circ} \Gamma_{1}$ is surjective, bounded, it induces the quasi-norm $\|\cdot\|_{\bar{B}^{\circ} \Gamma_{1} ; J}$ and $\pi: \ell_{p}\left(2^{-j m} G_{m}\right) \longrightarrow B_{j}^{\circ}$ is bounded for $j=0,1$. For $\bar{E}$ and $\bar{E}_{\Gamma_{2}}$ the relevant map is $\tau w=(\ldots, w, w, w, \ldots)$. Indeed, if we realize $\bar{E}_{\Gamma_{2}}$ as a $K$-space but replacing the $K$-functional by the equivalent $K_{r}$-functional, then $\tau$ is a metric injection from $\bar{E}_{\Gamma_{2}}$ into $\Gamma_{2}\left(W_{m}\right)$. Moreover, the restrictions $\tau: E_{j} \longrightarrow \ell_{\infty}\left(2^{-j m} W_{m}\right)$ are bounded for $j=0,1$. We have the following diagram

$$
\begin{gathered}
\ell_{p}\left(F_{m}\right) \times \ell_{p}\left(G_{m}\right) \stackrel{(\pi, \pi)}{\longrightarrow} A_{0}^{\circ} \times B_{0}^{\circ} \stackrel{T}{\longrightarrow} E_{0} \stackrel{\tau}{\longrightarrow} \ell_{\infty}\left(W_{m}\right) \\
\ell_{p}\left(2^{-m} F_{m}\right) \times \ell_{p}\left(2^{-m} G_{m}\right) \stackrel{(\pi, \pi)}{\longrightarrow} A_{1}^{\circ} \times B_{1}^{\circ} \stackrel{T}{\longrightarrow} E_{1} \stackrel{\tau}{\longrightarrow} \ell_{\infty}\left(2^{-m} W_{m}\right) \\
\Gamma_{0}\left(F_{m}\right) \times \Gamma_{1}\left(G_{m}\right) \stackrel{(\pi, \pi)}{\longrightarrow} \overline{A^{\circ}} \Gamma_{0} \times \overline{B^{\circ}} \Gamma_{1} \stackrel{T}{\longrightarrow} \bar{E}_{\Gamma_{2}} \stackrel{\tau}{\longrightarrow} \Gamma_{2}\left(W_{m}\right)
\end{gathered}
$$

Let $\widehat{T}=\tau T(\pi, \pi)$. The properties of the maps $\pi$ and $\tau$ yield that a necessary and sufficient condition for $T: \bar{A}^{\circ} \Gamma_{0} \times \bar{B}^{\circ} \Gamma_{1} \longrightarrow \bar{E}_{\Gamma_{2}}$ to be compact is that

$$
\widehat{T}: \Gamma_{0}\left(F_{m}\right) \times \Gamma_{1}\left(G_{m}\right) \longrightarrow \Gamma_{2}\left(W_{m}\right) \quad \text { is compact. }
$$


Since the sequences $\left(F_{m}\right),\left(G_{m}\right)$ are formed by $p$-normed quasi-Banach spaces, we have that the couples $\overline{\ell_{p}(F)}=\left(\ell_{p}\left(F_{m}\right), \ell_{p}\left(2^{-m} F_{m}\right)\right)$ and $\overline{\ell_{p}(G)}=\left(\ell_{p}\left(G_{m}\right), \ell_{p}\left(2^{-m} G_{m}\right)\right)$ are $p$-normed quasi-Banach couples. Moreover, the couple $\overline{\ell_{\infty}(W)}=\left(\ell_{\infty}\left(W_{m}\right), \ell_{\infty}\left(2^{-m} W_{m}\right)\right)$ is an $r$-normed quasi-Banach couple. These three couples are the relevant couples to work with $\widehat{T}$. In fact, the operator $\widehat{T}$ belongs to $\mathcal{B}\left(\overline{\ell_{p}(F)} \times \overline{\ell_{p}(G)}, \overline{\ell_{\infty}(W)}\right)$ and Lemma 2.4 shows that $\Gamma_{0}\left(F_{m}\right), \Gamma_{1}\left(G_{m}\right)$ and $\Gamma_{2}\left(W_{m}\right)$ are interpolation spaces with respect to the couples $\overline{\ell_{p}(F)}, \overline{\ell_{p}(G)}$ and $\overline{\ell_{\infty}(W)}$, respectively.

The following families of projections are useful in order to show the compactness of $\widehat{T}$ in (4.1). For $n \in \mathbb{N}$, put

$$
\begin{aligned}
& R_{n}\left(z_{m}\right)=\left(\ldots, 0,0, z_{-n}, z_{-n+1}, \ldots, z_{n-1}, z_{n}, 0,0, \ldots\right) \\
& R_{n}^{+}\left(z_{m}\right)=\left(\ldots, 0,0, z_{n+1}, z_{n+2}, z_{n+3}, \ldots\right) \\
& R_{n}^{-}\left(z_{m}\right)=\left(\ldots, z_{-n-3}, z_{-n-2}, z_{-n-1}, 0,0, \ldots\right)
\end{aligned}
$$

All these maps belong to $\mathcal{L}\left(\overline{\ell_{p}(F)}, \overline{\ell_{p}(F)}\right)$ and $R_{n} \in \mathcal{L}\left(\Sigma\left(\overline{\ell_{p}(F)}\right), \Delta\left(\overline{\ell_{p}(F)}\right)\right)$. One can easily check that they satisfy the following conditions:

(i) Each one of the maps $R_{n}, R_{n}^{+}, R_{n}^{-}$has quasi-norm 1 acting from $\ell_{p}\left(F_{m}\right)$ into $\ell_{p}\left(F_{m}\right)$, from $\ell_{p}\left(2^{-m} F_{m}\right)$ into $\ell_{p}\left(2^{-m} F_{m}\right)$ and from $\Gamma_{0}\left(F_{m}\right)$ into $\Gamma_{0}\left(F_{m}\right)$.

(ii) The identity operator $I$ on $\Sigma\left(\overline{\ell_{p}(F)}\right)$ can be decomposed as $I=R_{n}+R_{n}^{+}+R_{n}^{-}, n \in \mathbb{N}$.

(iii) For each $n \in \mathbb{N}$, projections $R_{n}: \ell_{p}\left(F_{m}\right) \longrightarrow \ell_{p}\left(2^{-m} F_{m}\right)$ and $R_{n}: \ell_{p}\left(2^{-m} F_{m}\right) \longrightarrow \ell_{p}\left(F_{m}\right)$ are bounded with

$$
\left\|R_{n}\right\|_{\ell_{p}\left(F_{m}\right), \ell_{p}\left(2^{-m} F_{m}\right)}=2^{n}=\left\|R_{n}\right\|_{\ell_{p}\left(2^{-m} F_{m}\right), \ell_{p}\left(F_{m}\right)} .
$$

Moreover $R_{n}^{+}: \ell_{p}\left(F_{m}\right) \longrightarrow \ell_{p}\left(2^{-m} F_{m}\right)$ and $R_{n}^{-}: \ell_{p}\left(2^{-m} F_{m}\right) \longrightarrow \ell_{p}\left(F_{m}\right)$ are also bounded with

$$
\left\|R_{n}^{+}\right\|_{\ell_{p}\left(F_{m}\right), \ell_{p}\left(2^{-m} F_{m}\right)}=2^{-(n+1)}=\left\|R_{n}^{-}\right\|_{\ell_{p}\left(2^{-m} F_{m}\right), \ell_{p}\left(F_{m}\right)} .
$$

Similar sequences of projections can be defined on the couples $\overline{\ell_{p}(G)}$ and $\overline{\ell_{\infty}(W)}$. We call them $S_{n}, S_{n}^{+}, S_{n}^{-}$ and $P_{n}, P_{n}^{+}, P_{n}^{-}$, respectively. They satisfy the corresponding versions of (i), (ii) and (iii).

Next we split the operator $\widehat{T}$ as in the Banach case [24, Theorem 3.1] and we work with each piece with the help of results of Section 3 and Theorems 4.1 and 4.2.

Using (ii), for $n \in \mathbb{N}$ we obtain $\widehat{T}=P_{n} \widehat{T}+P_{n}^{+} \widehat{T}+P_{n}^{-} \widehat{T}$. Moreover, $P_{n}^{-} \widehat{T}=P_{n}^{-} \widehat{T}\left(R_{n}+R_{n}^{+}+R_{n}^{-}, S_{n}+\right.$ $\left.S_{n}^{+}+S_{n}^{-}\right)$. Whence

$$
\begin{aligned}
\widehat{T} & =P_{n} \widehat{T}+P_{n}^{-} \widehat{T}\left(R_{n}, S_{n}\right)+P_{n}^{+} \widehat{T}+P_{n}^{-} \widehat{T}\left(R_{n}^{+}, S_{n}\right)+P_{n}^{-} \widehat{T}\left(R_{n}, S_{n}^{+}\right) \\
& +P_{n}^{-} \widehat{T}\left(R_{n}^{+}, S_{n}^{+}\right)+P_{n}^{-} \widehat{T}\left(R_{n}^{-}, S_{n}^{+}\right)+P_{n}^{-} \widehat{T}\left(R_{n}^{-}, S_{n}^{-}\right) \\
& +P_{n}^{-} \widehat{T}\left(R_{n}, S_{n}^{-}\right)+P_{n}^{-} \widehat{T}\left(R_{n}^{+}, S_{n}^{-}\right)+P_{n}^{-} \widehat{T}\left(R_{n}^{-}, S_{n}\right) .
\end{aligned}
$$

Suppose that $T: A_{1} \times B_{1} \longrightarrow E_{1}$ is compact. The case when we have compactness in $T: A_{0} \times B_{0} \longrightarrow E_{0}$ is similar. We are going to check that acting from $\Gamma_{0}\left(F_{m}\right) \times \Gamma_{1}\left(G_{m}\right)$ into $\Gamma_{2}\left(W_{m}\right)$ the operators $P_{n} \widehat{T}$ and $P_{n}^{-} \widehat{T}\left(R_{n}, S_{n}\right)$ are compact. Then we will show that the remaining nine operators have norms converging to zero as $n \rightarrow \infty$. This will show that $\widehat{T}$ in (4.1) is the limit of a sequence of compact bilinear operators and it is therefore compact. 
Having in mind the corresponding property to (iii) for $P_{n}$ and Lemma 2.4, we can factorize $P_{n} \widehat{T}$ by means of the diagram

$$
\ell_{p}\left(F_{m}\right) \times \ell_{p}\left(G_{m}\right) \quad \stackrel{\widehat{T}}{\longrightarrow} \ell_{\infty}\left(W_{m}\right) \stackrel{P_{n}}{\longrightarrow} \Delta\left(\overline{\ell_{\infty}(W)}\right) \hookrightarrow \Gamma_{2}\left(W_{m}\right) .
$$

We also have

$$
\ell_{p}\left(2^{-m} F_{m}\right) \times \ell_{p}\left(2^{-m} G_{m}\right) \quad \stackrel{\widehat{T}}{\longrightarrow} \ell_{\infty}\left(2^{-m} W_{m}\right) \stackrel{P_{n}}{\longrightarrow} \Delta\left(\overline{\ell_{\infty}(W)}\right) \hookrightarrow \Gamma_{2}\left(W_{m}\right)
$$

and this last operator is compact because compactness of $T: A_{1} \times B_{1} \longrightarrow E_{1}$ yields that $\widehat{T}: \ell_{p}\left(2^{-m} F_{m}\right) \times$ $\ell_{p}\left(2^{-m} G_{m}\right) \longrightarrow \ell_{\infty}\left(2^{-m} W_{m}\right)$ is compact. Using the diagram

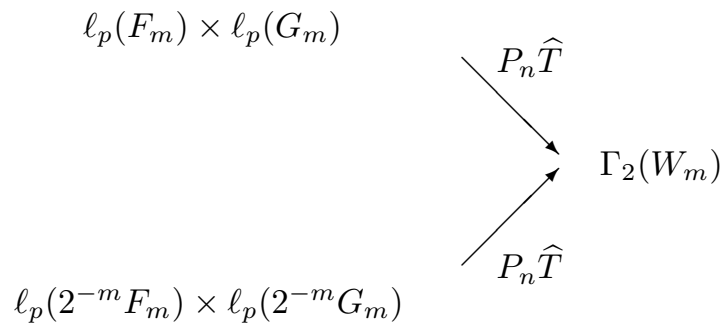

and applying Theorem 4.1 and Lemma 2.4, compactness of $P_{n} \widehat{T}: \Gamma_{0}\left(F_{m}\right) \times \Gamma_{1}\left(G_{m}\right) \longrightarrow \Gamma_{2}\left(W_{m}\right)$ follows.

Since

$$
\Gamma_{0}\left(F_{m}\right) \times \Gamma_{1}\left(G_{m}\right) \hookrightarrow \Sigma\left(\overline{\ell_{p}(F)}\right) \times \Sigma\left(\overline{\ell_{p}(G)}\right) \stackrel{\left(R_{n}, S_{n}\right)}{\longrightarrow} \Delta\left(\overline{\ell_{p}(F)}\right) \times \Delta\left(\overline{\ell_{p}(G)}\right)
$$

for the operator $P_{n}^{-} \widehat{T}\left(R_{n}, S_{n}\right)$ we can use the following diagram

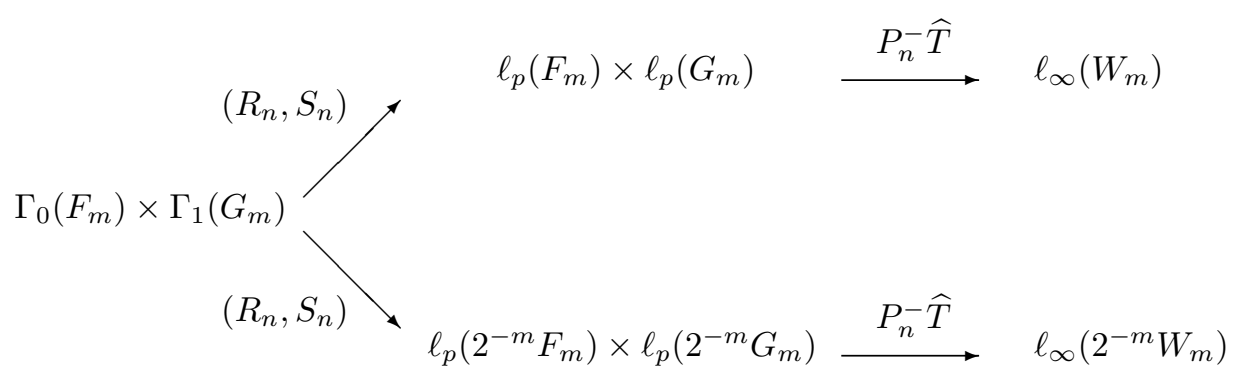

with $P_{n}^{-} \widehat{T}\left(R_{n}, S_{n}\right): \Gamma_{0}\left(F_{m}\right) \times \Gamma_{1}\left(G_{m}\right) \longrightarrow \ell_{\infty}\left(2^{-m} W_{m}\right)$ being compact. Whence, according to Theorem 4.2 and Lemma 2.4, we derive that $P_{n}^{-} \widehat{T}\left(R_{n}, S_{n}\right): \Gamma_{0}\left(F_{m}\right) \times \Gamma_{1}\left(G_{m}\right) \longrightarrow \Gamma_{2}\left(W_{m}\right)$ is also compact.

Next we show that the norm of $P_{n}^{+} \widehat{T}$ tends to 0 as $n \rightarrow \infty$. Consider the operator $P_{n}^{+} \tau T: A_{1}^{\circ} \times B_{1}^{\circ} \longrightarrow$ $\ell_{\infty}\left(2^{-m} W_{m}\right)$. Using the corresponding property to (iii) for $P_{n}^{+}$, given any $a \in \Delta(\bar{A})$ and $b \in \Delta(\bar{B})$, we have that

$$
\left\|P_{n}^{+} \tau T(a, b)\right\|_{\ell_{\infty}\left(2^{-m} W_{m}\right)} \leq 2^{-(n+1)}\|\tau T(a, b)\|_{\ell_{\infty}\left(W_{m}\right)} \rightarrow 0 \quad \text { as } \quad n \rightarrow \infty .
$$

Then, applying Lemma 3.2, we get that $\lim _{n \rightarrow \infty}\left\|P_{n}^{+} \tau T\right\|_{A_{1}^{\circ} \times B_{1}^{\circ}, \ell_{\infty}\left(2^{-m} W_{m}\right)}=0$ and consequently

$$
\lim _{n \rightarrow \infty}\left\|P_{n}^{+} \widehat{T}\right\|_{\ell_{p}\left(2^{-m} F_{m}\right) \times \ell_{p}\left(2^{-m} G_{m}\right), \ell_{\infty}\left(2^{-m} W_{m}\right)}=0 .
$$


Using now Theorem 3.1 and having in mind properties (2.3) and (2.2) of $f_{\Gamma_{1}}$, we obtain that

$$
\begin{aligned}
& \left\|P_{n}^{+} \widehat{T}\right\|_{\Gamma_{0}\left(F_{m}\right) \times \Gamma_{1}\left(G_{m}\right), \Gamma_{2}\left(W_{m}\right)} \\
& \leq C_{1}\left\|P_{n}^{+} \widehat{T}\right\|_{\ell_{p}\left(F_{m}\right) \times \ell_{p}\left(G_{m}\right), \ell_{\infty}\left(W_{m}\right)} f_{\Gamma_{1}}\left(\frac{\left\|P_{n}^{+} \widehat{T}\right\|_{\ell_{p}\left(2^{-m} F_{m}\right) \times \ell_{p}\left(2^{-m} G_{m}\right), \ell_{\infty}\left(2^{-m} W_{m}\right)}}{\left\|P_{n}^{+} \widehat{T}\right\|_{\ell_{p}\left(F_{m}\right) \times \ell_{p}\left(G_{m}\right), \ell_{\infty}\left(W_{m}\right)}}\right) \\
& \leq C_{2}\left\|P_{n}^{+} \widehat{T}\right\|_{\ell_{p}\left(F_{m}\right) \times \ell_{p}\left(G_{m}\right), \ell_{\infty}\left(W_{m}\right)} f_{\Gamma_{1}}\left(\frac{1}{\left\|P_{n}^{+} \widehat{T}\right\|_{\ell_{p}\left(F_{m}\right) \times \ell_{p}\left(G_{m}\right), \ell_{\infty}\left(W_{m}\right)}}\right) \\
& \quad \times f_{\Gamma_{1}}\left(\left\|P_{n}^{+} \widehat{T}\right\|_{\ell_{p}\left(2^{-m} F_{m}\right) \times \ell_{p}\left(2^{-m} G_{m}\right), \ell_{\infty}\left(2^{-m} W_{m}\right)}\right) \\
& \leq C_{3} f_{\Gamma_{1}}\left(\left\|P_{n}^{+} \widehat{T}\right\|_{\ell_{p}\left(2^{-m} F_{m}\right) \times \ell_{p}\left(2^{-m} G_{m}\right), \ell_{\infty}\left(2^{-m} W_{m}\right)}\right) \longrightarrow 0 \text { when } \quad n \rightarrow \infty .
\end{aligned}
$$

For the operator $P_{n}^{-} \widehat{T}\left(R_{n}^{+}, S_{n}\right)$, factorization

$$
\ell_{p}\left(F_{m}\right) \times \ell_{p}\left(G_{m}\right) \stackrel{\left(R_{n}^{+}, S_{n}\right)}{\longrightarrow} \ell_{p}\left(2^{-m} F_{m}\right) \times \ell_{p}\left(2^{-m} G_{m}\right) \stackrel{\widehat{T}}{\longrightarrow} \ell_{\infty}\left(2^{-m} W_{m}\right) \stackrel{P_{n}^{-}}{\longrightarrow} \ell_{\infty}\left(W_{m}\right)
$$

and (iii) give that

$$
\begin{aligned}
& \left\|P_{n}^{-} \widehat{T}\left(R_{n}^{+}, S_{n}\right)\right\|_{\ell_{p}\left(F_{m}\right) \times \ell_{p}\left(G_{m}\right), \ell_{\infty}\left(W_{m}\right)} \\
& \leq\left\|R_{n}^{+}\right\|_{\ell_{p}\left(F_{m}\right), \ell_{p}\left(2^{-m} F_{m}\right)}\left\|S_{n}\right\|_{\ell_{p}\left(G_{m}\right), \ell_{p}\left(2^{-m} G_{m}\right)} \\
& \times\|\widehat{T}\|_{\ell_{p}\left(2^{-m} F_{m}\right) \times \ell_{p}\left(2^{-m} G_{m}\right), \ell_{\infty}\left(2^{-m} W_{m}\right)}\left\|P_{n}^{-}\right\|_{\ell_{\infty}\left(2^{-m} W_{m}\right), \ell_{\infty}\left(W_{m}\right)} \\
& \leq 2^{-(n+1)} 2^{n}\|T\|_{A_{1}^{\circ} \times B_{1}^{\circ}, E_{1}} 2^{-(n+1)} \longrightarrow 0 \quad \text { as } \quad n \rightarrow \infty \text {. }
\end{aligned}
$$

On the other hand,

$$
\begin{aligned}
& \left\|P_{n}^{-} \widehat{T}\left(R_{n}^{+}, S_{n}\right)\right\|_{\ell_{p}\left(2^{-m} F_{m}\right) \times \ell_{p}\left(2^{-m} G_{m}\right), \ell_{\infty}\left(2^{-m} W_{m}\right)} \\
& \leq\left\|R_{n}^{+}\right\|_{\ell_{p}\left(2^{-m} F_{m}\right), \ell_{p}\left(2^{-m} F_{m}\right)}\left\|S_{n}\right\|_{\ell_{p}\left(2^{-m} G_{m}\right), \ell_{p}\left(2^{-m} G_{m}\right)} \\
& \times\|\widehat{T}\|_{\ell_{p}\left(2^{-m} F_{m}\right) \times \ell_{p}\left(2^{-m} G_{m}\right), \ell_{\infty}\left(2^{-m} W_{m}\right)}\left\|P_{n}^{-}\right\|_{\ell_{\infty}\left(2^{-m} W_{m}\right), \ell_{\infty}\left(2^{-m} W_{m}\right)} \\
& \leq\|T\|_{A_{1}^{\circ} \times B_{1}^{\circ}, E_{1}} \text {. }
\end{aligned}
$$

Hence, it follows from Theorem 3.1 and properties (2.3) and (2.2) of $f_{\Gamma_{1}}$ that

$$
\lim _{n \rightarrow \infty}\left\|P_{n}^{-} \widehat{T}\left(R_{n}^{+}, S_{n}\right)\right\|_{\Gamma_{0}\left(F_{m}\right) \times \Gamma_{1}\left(G_{m}\right), \Gamma_{2}\left(W_{m}\right)}=0 .
$$

Operators $P_{n}^{-} \widehat{T}\left(R_{n}, S_{n}^{+}\right)$and $P_{n}^{-} \widehat{T}\left(R_{n}^{+}, S_{n}^{+}\right)$can be treated similarly.

Next we consider the operator $P_{n}^{-} \widehat{T}\left(R_{n}^{-}, S_{n}^{+}\right)=P_{n}^{-} \tau T\left(\pi R_{n}^{-}, \pi S_{n}^{+}\right)$. For the quasi-norm of $T\left(\pi R_{n}^{-}, \pi S_{n}^{+}\right)$ acting from $\ell_{p}\left(2^{-m} F_{m}\right) \times \ell_{p}\left(2^{-m} G_{m}\right)$ into $E_{0}+E_{1}$ we have that

$$
\begin{aligned}
& \left\|T\left(\pi R_{n}^{-}, \pi S_{n}^{+}\right)\right\|_{\ell_{p}\left(2^{-m} F_{m}\right) \times \ell_{p}\left(2^{-m} G_{m}\right), \Sigma(\bar{E})} \\
& \leq\|T\|_{\Sigma\left(\overline{A^{\circ}}\right) \times \Sigma\left(\overline{B^{\circ}}\right), \Sigma(\bar{E})}\left\|\pi R_{n}^{-}\right\|_{\ell_{p}\left(2^{-m} F_{m}\right), \Sigma\left(\overline{A^{\circ}}\right)}\left\|\pi S_{n}^{+}\right\|_{\ell_{p}\left(2^{-m} G_{m}\right), \Sigma\left(\overline{B^{\circ}}\right)} \\
& \leq\|T\|_{\Sigma\left(\overline{A^{\circ}}\right) \times \Sigma\left(\overline{B^{\circ}}\right), \Sigma(\bar{E})}\|\pi\|_{\ell_{p}\left(F_{m}\right), \Sigma\left(\overline{A^{\circ}}\right)}\left\|R_{n}^{-}\right\|_{\ell_{p}\left(2^{-m} F_{m}\right), \ell_{p}\left(F_{m}\right)} \\
& \quad \times\|\pi\|_{\ell_{p}\left(2^{-m} G_{m}\right), \Sigma\left(\overline{B^{\circ}}\right)}\left\|S_{n}^{+}\right\|_{\ell_{p}\left(2^{-m} G_{m}\right), \ell_{p}\left(2^{-m} G_{m}\right)} \\
& \leq 2^{-(n+1)}\|T\|_{\Sigma\left(\overline{A^{\circ}}\right) \times \Sigma\left(\overline{B^{\circ}}\right), \Sigma(\bar{E})}^{\longrightarrow} \quad \text { as } n \rightarrow \infty .
\end{aligned}
$$

Since $T: A_{1}^{\circ} \times B_{1}^{\circ} \longrightarrow E_{1}$ is compact, Lemma 3.3 yields that

$$
\lim _{n \rightarrow \infty}\left\|T\left(\pi R_{n}^{-}, \pi S_{n}^{+}\right)\right\|_{\ell_{p}\left(2^{-m} F_{m}\right) \times \ell_{p}\left(2^{-m} G_{m}\right), E_{1}}=0 .
$$


Therefore, $\lim _{n \rightarrow \infty}\left\|P_{n}^{-} \widehat{T}\left(R_{n}^{-}, S_{n}^{+}\right)\right\|_{\ell_{p}\left(2^{-m} F_{m}\right) \times \ell_{p}\left(2^{-m} G_{m}\right), \ell_{\infty}\left(2^{-m} W_{m}\right)}=0$. For the norm of the other restriction we get $\left\|P_{n}^{-} \widehat{T}\left(R_{n}^{-}, S_{n}^{+}\right)\right\|_{\ell_{p}\left(F_{m}\right) \times \ell_{p}\left(G_{m}\right), \ell_{\infty}\left(W_{m}\right)} \leq\|T\|_{A_{0}^{\circ} \times B_{0}^{\circ}, E_{0}}$. Consequently, using again Theorem 3.1 and the properties of $f_{\Gamma_{1}}$ we derive that

$$
\lim _{n \rightarrow \infty}\left\|P_{n}^{-} \widehat{T}\left(R_{n}^{-}, S_{n}^{+}\right)\right\|_{\Gamma_{0}\left(F_{m}\right) \times \Gamma_{1}\left(G_{m}\right), \Gamma_{2}\left(W_{m}\right)}=0 .
$$

For the remaining operators $P_{n}^{-} \widehat{T}\left(R_{n}^{-}, S_{n}^{-}\right), P_{n}^{-} \widehat{T}\left(R_{n}, S_{n}^{-}\right), P_{n}^{-} \widehat{T}\left(R_{n}^{+}, S_{n}^{-}\right)$and $P_{n}^{-} \widehat{T}\left(R_{n}^{-}, S_{n}\right)$ we can proceed similarly as with $P_{n}^{-} \widehat{T}\left(R_{n}^{-}, S_{n}^{+}\right)$and show that their norms converge also to 0 as $n \rightarrow \infty$. This completes the proof.

For the Banach case, that is, when $\bar{A}, \bar{B}, \bar{E}$ are Banach couples, $p=r=1$ and $\Gamma_{0}, \Gamma_{1}, \Gamma_{2}$ are Banach sequence lattices satisfying (2.1), (2.6) and (3.1), then Theorem 4.7 recovers [24, Theorem 3.1]. In particular, in the Banach case Theorem 4.7 improves [23, Theorem 5.8].

Applying Theorem 4.7 to the case of the real method with a function parameter described in Example 2.5, we get the following result.

Theorem 4.8 Let $\bar{A}=\left(A_{0}, A_{1}\right), \bar{B}=\left(B_{0}, B_{1}\right)$ be quasi-Banach couples and let $\bar{E}=\left(E_{0}, E_{1}\right)$ be an $r$ normed quasi-Banach couple $(0<r \leq 1)$. Suppose that $\rho_{0}, \rho_{1}, \rho_{2}$ are function parameters such that for some constant $C>0$ we have

$$
\rho_{0}(t) \rho_{1}(s) \leq C \rho_{2}(t s) \quad t, s>0 .
$$

Let $0<q_{0}, q_{1} \leq \infty$ and write

$$
\frac{1}{q}=\left\{\begin{array}{lll}
\frac{1}{q_{0}}+\frac{1}{q_{1}}-\frac{1}{r} & \text { if } & q_{0}, q_{1} \geq r, \\
\frac{1}{\max \left(q_{0}, q_{1}\right)} & \text { if } & q_{0}<r \text { or } q_{1}<r .
\end{array}\right.
$$

If $T: \bar{A} \times \bar{B} \longrightarrow \bar{E}$ and $T: A_{j} \times B_{j} \longrightarrow E_{j}$ is compact for $j=0$ or 1 , then

$$
T:\left(A_{0}, A_{1}\right)_{\rho_{0}, q_{0}} \times\left(B_{0}, B_{1}\right)_{\rho_{1}, q_{1}} \longrightarrow\left(E_{0}, E_{1}\right)_{\rho_{2}, q}
$$

is also compact.

Proof. Assume first that $q_{0}, q_{1} \geq r$, so $r / q+1=r / q_{0}+r / q_{1}$. If $\xi=\left(\xi_{m}\right) \in \ell_{q_{0}}\left(1 / \rho_{0}\left(2^{m}\right)\right), \eta=\left(\eta_{m}\right) \in$ $\ell_{q_{1}}\left(1 / \rho_{1}\left(2^{m}\right)\right)$ are non-negative scalar sequences, then according to (4.2) and Young's inequality we obtain

$$
\begin{aligned}
\|\left(\xi^{r} \star \eta^{r}\right)^{1 / r} & \|_{\ell_{q}\left(1 / \rho_{2}\left(2^{m}\right)\right)}=\left(\sum_{m=-\infty}^{\infty}\left(\sum_{k=-\infty}^{\infty} \xi_{k}^{r} \eta_{m-k}^{r} / \rho_{2}\left(2^{m}\right)^{r}\right)^{q / r}\right)^{1 / q} \\
& \leq C\left(\sum_{m=-\infty}^{\infty}\left(\sum_{k=-\infty}^{\infty}\left(\xi_{k} / \rho_{0}\left(2^{k}\right)\right)^{r}\left(\eta_{m-k} / \rho_{1}\left(2^{m-k}\right)\right)^{r}\right)^{q / r}\right)^{1 / q} \\
& \leq C\left\|\left(\xi_{m} / \rho_{0}\left(2^{m}\right)\right)^{r}\right\|_{\ell_{q_{0} / r}}^{1 / r}\left\|\left(\eta_{m} / \rho_{1}\left(2^{m}\right)\right)^{r}\right\|_{\ell_{q_{1} / r}}^{1 / r} \\
& =C\|\xi\|_{\ell_{q_{0}}\left(1 / \rho_{0}\left(2^{m}\right)\right)}\|\eta\|_{\ell_{q_{1}}\left(1 / \rho_{1}\left(2^{m}\right)\right)} .
\end{aligned}
$$

This shows that inequality (3.1) holds. Hence, the result follows from Theorem 4.7.

Suppose now that $q_{0}<r$ or $q_{1}<r$. Then either

(a) $\quad q_{1}=\max \left(q_{0}, q_{1}\right)=q \quad$ and $\quad q_{0}<r$, 
or

$$
q_{0}=\max \left(q_{0}, q_{1}\right)=q \quad \text { and } \quad q_{1}<r
$$

If (a) holds, then $1 / q=1 / q_{0}+1 / q_{1}-1 / q_{0}$. Moreover, since the couple $\left(E_{0}, E_{1}\right)$ is $r$-normed, it is also $q_{0^{-}}$ normed. Therefore, we are in the situation considered before and the result follows. If (b) holds, we can proceed similarly.

If we choose $\rho_{0}(t)=\rho_{1}(t)=\rho_{2}(t)=t^{\theta}$ with $0<\theta<1$ then we get the following result for the real method.

Theorem 4.9 Let $\bar{A}=\left(A_{0}, A_{1}\right), \bar{B}=\left(B_{0}, B_{1}\right)$ be quasi-Banach couples and let $\bar{E}=\left(E_{0}, E_{1}\right)$ be an $r$ normed quasi-Banach couple $(0<r \leq 1)$. Let $0<\theta<1,0<q_{0}, q_{1} \leq \infty$ and let $0<q \leq \infty$ satisfying that

$$
\frac{1}{q}=\left\{\begin{array}{lll}
\frac{1}{q_{0}}+\frac{1}{q_{1}}-\frac{1}{r} & \text { if } \quad q_{0}, q_{1} \geq r \\
\frac{1}{\max \left(q_{0}, q_{1}\right)} & \text { if } \quad q_{0}<\operatorname{ror} q_{1}<r
\end{array}\right.
$$

If $T: \bar{A} \times \bar{B} \longrightarrow \bar{E}$ and $T: A_{j} \times B_{j} \longrightarrow E_{j}$ is compact for $j=0$ or 1 , then

$$
T:\left(A_{0}, A_{1}\right)_{\theta, q_{0}} \times\left(B_{0}, B_{1}\right)_{\theta, q_{1}} \longrightarrow\left(E_{0}, E_{1}\right)_{\theta, q}
$$

is compact as well.

Remark 4.10 Assumption $T: \bar{A} \times \bar{B} \longrightarrow \bar{E}$ implies that

$$
T \in \mathcal{B}(\Sigma(\bar{A}) \times \Sigma(\bar{B}), \Sigma(\bar{E})) \text { and that } T \in \mathcal{B}\left(A_{j} \times B_{j}, E_{j}\right), j=0,1 \text {. }
$$

Hence, there is a constant $M>0$ such that

$$
\|T(a, b)\|_{E_{j}} \leq M\|a\|_{A_{j}}\|b\|_{B_{j}}, a \in \Delta(\bar{A}), b \in \Delta(\bar{B}), j=0,1 .
$$

In applications sometimes we have (4.3) but we do not have that $T \in \mathcal{B}(\Sigma(\bar{A}) \times \Sigma(\bar{B}), \Sigma(\bar{E}))$. However, if we have (4.3) and some extra information on the operator $T$ then there is a certain replacement for the assumption $T \in \mathcal{B}(\Sigma(\bar{A}) \times \Sigma(\bar{B}), \Sigma(\bar{E}))$. Next we show it.

Suppose that (4.3) holds. Let $a \in \Delta(\bar{A}), b \in \Delta(\bar{B})$ with $b=b_{0}+b_{1}$ and $b_{j} \in B_{j}, j=0,1$, then $b_{j} \in \Delta(\bar{B})$ and so

$$
\|T(a, b)\|_{\Sigma(\bar{E})} \leq\left\|T\left(a, b_{0}\right)\right\|_{E_{0}}+\left\|T\left(a, b_{1}\right)\right\|_{E_{1}} \leq M J(1, a)\left(\left\|b_{0}\right\|_{B_{0}}+\left\|b_{1}\right\|_{B_{1}}\right)
$$

This yields that

$$
\|T(a, b)\|_{\Sigma(\bar{E})} \leq M\|a\|_{A_{0} \cap A_{1}}\|b\|_{B_{0}+B_{1}}
$$

If $\Delta(\bar{B})$ is dense in $B_{0}$ and $B_{1}$, then $T$ may be uniquely extended to a bounded bilinear operator

$$
T: \Delta(\bar{A}) \times \Sigma(\bar{B}) \longrightarrow \Sigma(\bar{E})
$$

Similarly, if $\Delta(\bar{A})$ is dense in $A_{0}$ and $A_{1}$, it follows from (4.3) that $T$ has a unique extension to a bounded bilinear operator

$$
T: \Sigma(\bar{A}) \times \Delta(\bar{B}) \longrightarrow \Sigma(\bar{E}) .
$$


Next we follow an idea of Janson [29]. Let $0<\theta_{j}, \mu_{j}<1,0<q_{j}, r_{j}<\infty, j=0,1,2$. Suppose that the operator $T$ satisfies that

$$
\|T(a, b)\|_{\bar{E}_{\theta_{2}, q_{2}}} \leq M_{1}\|a\|_{\bar{A}_{\theta_{0}, q_{0}}}\|b\|_{\bar{B}_{\theta_{1}, q_{1}}}, a \in \Delta(\bar{A}), b \in \Delta(\bar{B})
$$

and

$$
\|T(a, b)\|_{\bar{E}_{\mu_{2}, r_{2}}} \leq M_{2}\|a\|_{\bar{A}_{\mu_{0}, r_{0}}}\|b\|_{\bar{B}_{\mu_{1}, r_{1}}}, a \in \Delta(\bar{A}), b \in \Delta(\bar{B}) .
$$

Estimates (4.5) and (4.6) may be deduced from (4.3) by means of the bilinear interpolation theorem for the real method provided parameters $\theta_{j}, \mu_{j}, q_{j}, r_{j}$ satisfy suitable conditions. Assume in addition that we have the following extra information on $T$

$$
\begin{aligned}
& \|T(a, b)\|_{E_{0}+E_{1}} \leq M_{3}\|a\|_{\bar{A}_{\theta_{0}, q_{0}}}\|b\|_{\bar{B}_{\mu_{1}, r_{1}}}, a \in \Delta(\bar{A}), b \in \Delta(\bar{B}), \\
& \|T(a, b)\|_{E_{0}+E_{1}} \leq M_{4}\|a\|_{\bar{A}_{\mu_{0}, r_{0}}}\|b\|_{\bar{B}_{\theta_{1}, q_{1}}}, a \in \Delta(\bar{A}), b \in \Delta(\bar{B}) .
\end{aligned}
$$

Since $q_{j}, r_{j}<\infty$, then $\Delta(\bar{A})$ is dense in $\bar{A}_{\theta_{0}, q_{0}}$ and $\bar{A}_{\mu_{0}, r_{0}}$ (see [4, Theorem 3.4.2/(b) and page 66]), and $\Delta(\bar{B})$ is dense in $\bar{B}_{\theta_{1}, q_{1}}$ and $\bar{B}_{\mu_{1}, r_{1}}$. Proceeding as we have done to established (4.4), it follows from (4.5) and (4.7) that $T$ may be uniquely extended to a bounded bilinear operator

$$
T: \bar{A}_{\theta_{0}, q_{0}} \times\left(\bar{B}_{\theta_{1}, q_{1}}+\bar{B}_{\mu_{1}, r_{1}}\right) \longrightarrow E_{0}+E_{1} .
$$

On the other hand, by (4.6) and (4.8), $T$ has a unique extension to a bounded bilinear operator

$$
T: \bar{A}_{\mu_{0}, r_{0}} \times\left(\bar{B}_{\theta_{1}, q_{1}}+\bar{B}_{\mu_{1}, r_{1}}\right) \longrightarrow E_{0}+E_{1}
$$

Finally, from (4.9) and (4.10), it follows that $T$ may be uniquely extended to a bounded bilinear operator

$$
T:\left(\bar{A}_{\theta_{0}, q_{0}}+\bar{A}_{\mu_{0}, r_{0}}\right) \times\left(\bar{B}_{\theta_{1}, q_{1}}+\bar{B}_{\mu_{1}, r_{1}}\right) \longrightarrow E_{0}+E_{1},
$$

which may be used as a replacement for the assumption $T \in \mathcal{B}(\Sigma(\bar{A}) \times \Sigma(\bar{B}), \Sigma(\bar{E}))$.

\section{Compact bilinear operators among $L_{p}$ spaces}

Let $(\Omega, \mu)$ be a $\sigma$-finite measure space. We denote by $\mathcal{M}(\mu)$ the collection of all (equivalence classes of) measurable functions $f$ on $\Omega$ which are finite almost everywhere. We endow $\mathcal{M}(\mu)$ with the topology of convergence in measure on each measurable set of finite measure. In this way, $\mathcal{M}(\mu)$ is a metrizable topological vector space.

For $0<p \leq \infty$, we let $L_{p}(\Omega)$ be the usual Lebesgue space. Given $0<p<\infty$ and $0<q \leq \infty$, the Lorentz space $L_{p, q}(\Omega)$ is defined to be the set of all (equivalence classes of) measurable functions $f$ on $\Omega$ which have a finite quasi-norm

$$
\|f\|_{L_{p, q}(\Omega)}=\left(\int_{0}^{\mu(\Omega)}\left(t^{1 / p} f^{*}(t)\right)^{q} \frac{d t}{t}\right)^{1 / q}
$$

(the integral should be replaced by the supremum if $q=\infty$ ). Here $f^{*}$ stands for the non-increasing rearrangement of $f$. When $p=q$ we have $L_{p}(\Omega)=L_{p, p}(\Omega)$. The Lebesgue spaces $L_{p}(\Omega)$ and the Lorentz spaces $L_{p, q}(\Omega)$ are continuously embedded in $\mathcal{M}(\mu)$.

If $0<p<\infty$ it turns out that

$$
K\left(t, f ; L_{p}(\Omega), L_{\infty}(\Omega)\right) \sim\left(\int_{0}^{t^{p}}\left(f^{*}(t)\right)^{p} d t\right)^{1 / p} .
$$


Moreover, for $0<q \leq \infty, 0<r_{0} \neq r_{1} \leq \infty, 0<\theta<1$ and $1 / r=(1-\theta) / r_{0}+\theta / r_{1}$, then we have

$$
\left(L_{r_{0}}(\Omega), L_{r_{1}}(\Omega)\right)_{\theta, q}=L_{r, q}(\Omega) \quad \text { (equivalent quasi-norms) }
$$

(see [4, Theorems 5.2.1 and 5.3.1] or [44, 1.18.6]).

The following interpolation result is a consequence of Theorem $B_{1}$ in the paper [8] by Calderón and Zygmund.

Theorem 5.1 Let $\left(\Omega_{k}, \mu_{k}\right)$ be $\sigma$-finite measure spaces for $k=0,1,2$. Suppose $1 \leq p_{j}, q_{j} \leq \infty$ and $0<$ $r_{j} \leq \infty, j=0,1$. Let $0<\theta<1$ and put $1 / p=(1-\theta) / p_{0}+\theta / p_{1}, 1 / q=(1-\theta) / q_{0}+\theta / q_{1}$ and $1 / r=(1-\theta) / r_{0}+\theta / r_{1}$. Assume that $p \neq \infty, q \neq \infty$ and let

$$
T:\left(L_{p_{0}}\left(\Omega_{0}\right)+L_{p_{1}}\left(\Omega_{0}\right)\right) \times\left(L_{q_{0}}\left(\Omega_{1}\right)+L_{q_{1}}\left(\Omega_{1}\right)\right) \longrightarrow\left(L_{r_{0}}\left(\Omega_{2}\right)+L_{r_{1}}\left(\Omega_{2}\right)\right)
$$

be a bounded bilinear operator such that for $j=0,1$ the restriction

$$
T: L_{p_{j}}\left(\Omega_{0}\right) \times L_{q_{j}}\left(\Omega_{1}\right) \longrightarrow L_{r_{j}}\left(\Omega_{2}\right)
$$

is bounded with quasi-norm $M_{j}$. Then

$$
T: L_{p}\left(\Omega_{0}\right) \times L_{q}\left(\Omega_{1}\right) \longrightarrow L_{r}\left(\Omega_{2}\right)
$$

is also bounded with quasi-norm $M \leq M_{0}^{1-\theta} M_{1}^{\theta}$.

Next we are going to establish a reinforced version of this result.

If $D \subseteq \Omega_{2}$ is a $\mu_{2}$-measurable set, we put $P_{D}$ for the linear operator defined by $P_{D} f=\chi_{D} f$.

Theorem 5.2 Let $\left(\Omega_{k}, \mu_{k}\right)$ be $\sigma$-finite measure spaces for $k=0,1,2$. Suppose $1 \leq p_{j}, q_{j} \leq \infty$ and $0<$ $r_{j} \leq \infty, j=0,1$. Let $0<\theta<1$ and put $1 / p=(1-\theta) / p_{0}+\theta / p_{1}, 1 / q=(1-\theta) / q_{0}+\theta / q_{1}$ and $1 / r=(1-\theta) / r_{0}+\theta / r_{1}$. Suppose that $p \neq \infty, q \neq \infty$ and let

$$
T:\left(L_{p_{0}}\left(\Omega_{0}\right)+L_{p_{1}}\left(\Omega_{0}\right)\right) \times\left(L_{q_{0}}\left(\Omega_{1}\right)+L_{q_{1}}\left(\Omega_{1}\right)\right) \longrightarrow L_{r_{0}}\left(\Omega_{2}\right)+L_{r_{1}}\left(\Omega_{2}\right)
$$

be a bounded bilinear operator such that for $j=0,1$ the restriction

$$
T: L_{p_{j}}\left(\Omega_{0}\right) \times L_{q_{j}}\left(\Omega_{1}\right) \longrightarrow L_{r_{j}}\left(\Omega_{2}\right)
$$

is bounded. Assume, in addition, that $r_{0} \neq \infty$ and that

$$
T: L_{p_{0}}\left(\Omega_{0}\right) \times L_{q_{0}}\left(\Omega_{1}\right) \longrightarrow L_{r_{0}}\left(\Omega_{2}\right) \text { is compact }
$$

Then

$$
T: L_{p}\left(\Omega_{0}\right) \times L_{q}\left(\Omega_{1}\right) \longrightarrow L_{r}\left(\Omega_{2}\right) \quad \text { is also compact } .
$$

Pro of. Let $U_{L_{p}}$ be the closed unit ball of $L_{p}$ and let $U_{L_{q}}$ be the corresponding ball in $L_{q}$. Our aim is to show that $T\left(U_{L_{p}}, U_{L_{q}}\right)=\left\{T(f, g): f \in U_{L_{p}}, g \in U_{L_{q}}\right\}$ is relatively compact set in $L_{r}\left(\Omega_{2}\right)$. Since $r_{0}<\infty$, we also have $r<\infty$. Then, according to [35, Lemma I.1.1] or [1, page 31], the set $T\left(U_{L_{p}}, U_{L_{q}}\right)$ is relatively compact in $L_{r}\left(\Omega_{2}\right)$ if, and only if, the following two properties hold:

(a) $\lim _{\mu_{2}(D) \rightarrow 0}\left\|P_{D} T\right\|_{L_{p} \times L_{q}, L_{r}}=0$.

(b) $T\left(U_{L_{p}}, U_{L_{q}}\right)$ is relatively compact in $\mathcal{M}\left(\mu_{2}\right)$. 
Let $D \subseteq \Omega_{2}$ be any $\mu_{2}$-measurable set. Since $\left\|P_{D} T f\right\|_{L_{r_{j}}} \leq\|T f\|_{L_{r_{j}}}$, we have that

$$
P_{D} T:\left(L_{p_{0}}\left(\Omega_{0}\right), L_{p_{1}}\left(\Omega_{0}\right)\right) \times\left(L_{q_{0}}\left(\Omega_{1}\right), L_{q_{1}}\left(\Omega_{1}\right)\right) \longrightarrow\left(L_{r_{0}}\left(\Omega_{2}\right), L_{r_{1}}\left(\Omega_{2}\right)\right) .
$$

Moreover $\lim _{\mu_{2}(D) \rightarrow 0}\left\|P_{D} T\right\|_{L_{p_{0}} \times L_{q_{0}}, L_{r_{0}}}=0$ because $T: L_{p_{0}}\left(\Omega_{0}\right) \times L_{q_{0}}\left(\Omega_{1}\right) \longrightarrow L_{r_{0}}\left(\Omega_{2}\right)$ is compact. Hence, using Theorem 5.1, we derive that

$$
\begin{aligned}
\left\|P_{D} T\right\|_{L_{p} \times L_{q}, L_{r}} & \leq\left\|P_{D} T\right\|_{L_{p_{0}} \times L_{q_{0}}, L_{r_{0}}}^{1-\theta}\left\|P_{D} T\right\|_{L_{p_{1}} \times L_{q_{1}}, L_{r_{1}}}^{\theta} \\
& \leq\left\|P_{D} T\right\|_{L_{p_{0}} \times L_{q_{0}}, L_{r_{0}}}^{1-\theta}\|T\|_{L_{p_{1}} \times L_{q_{1}}, L_{r_{1}}}^{\theta} \longrightarrow 0 \text { as } \mu_{2}(D) \rightarrow 0 .
\end{aligned}
$$

This establishes (a).

In order to check (b), take $0<\varepsilon<1$ and let $s=\min \left(r_{0}, r_{1}, 1, \varepsilon p, \varepsilon q\right)$. Then the couple $\left(L_{r_{0}}\left(\Omega_{2}\right), L_{r_{1}}\left(\Omega_{2}\right)\right)$ is $s$-normed. Whence, Theorem 4.9 yields that the restrictions

$$
\begin{aligned}
& T:\left(L_{p_{0}}\left(\Omega_{0}\right), L_{p_{1}}\left(\Omega_{0}\right)\right)_{\theta, s} \times\left(L_{q_{0}}\left(\Omega_{1}\right), L_{q_{1}}\left(\Omega_{1}\right)\right)_{\theta, \infty} \longrightarrow\left(L_{r_{0}}\left(\Omega_{2}\right), L_{r_{1}}\left(\Omega_{2}\right)\right)_{\theta, \infty} \\
& T:\left(L_{p_{0}}\left(\Omega_{0}\right), L_{p_{1}}\left(\Omega_{0}\right)\right)_{\theta, \infty} \times\left(L_{q_{0}}\left(\Omega_{1}\right), L_{q_{1}}\left(\Omega_{1}\right)\right)_{\theta, s} \longrightarrow\left(L_{r_{0}}\left(\Omega_{2}\right), L_{r_{1}}\left(\Omega_{2}\right)\right)_{\theta, \infty}
\end{aligned}
$$

are compact. The target space being

$$
\left(L_{r_{0}}\left(\Omega_{2}\right), L_{r_{1}}\left(\Omega_{2}\right)\right)_{\theta, \infty}=\left\{\begin{array}{lll}
L_{r, \infty}\left(\Omega_{2}\right) & \text { if } & r_{0} \neq r_{1}, \\
L_{r}\left(\Omega_{2}\right) & \text { if } & r_{0}=r_{1}=r .
\end{array}\right.
$$

Choose $0<\eta_{0}, \eta_{1}<1$ such that $1 / p=\left(1-\eta_{0}\right) / s$ and $1 / q=\eta_{1} / s$. According to [4, Theorem 5.2.4], we get

$$
\left(\left(L_{p_{0}}\left(\Omega_{0}\right), L_{p_{1}}\left(\Omega_{0}\right)\right)_{\theta, s},\left(L_{p_{0}}\left(\Omega_{0}\right), L_{p_{1}}\left(\Omega_{0}\right)\right)_{\theta, \infty}\right)_{\eta_{0}, p}=\left(L_{p_{0}}\left(\Omega_{0}\right), L_{p_{1}}\left(\Omega_{0}\right)\right)_{\theta, p}=L_{p}\left(\Omega_{0}\right)
$$

and

$$
\left(\left(L_{q_{0}}\left(\Omega_{1}\right), L_{q_{1}}\left(\Omega_{1}\right)\right)_{\theta, \infty},\left(L_{q_{0}}\left(\Omega_{1}\right), L_{q_{1}}\left(\Omega_{1}\right)\right)_{\theta, s}\right)_{\eta_{1}, q}=\left(L_{q_{0}}\left(\Omega_{1}\right), L_{q_{1}}\left(\Omega_{1}\right)\right)_{\theta, q}=L_{q}\left(\Omega_{1}\right) .
$$

Now applying Corollary 4.5 to restrictions (5.1) and (5.2) and having in mind the previous reiteration formulae, we conclude that $T: L_{p}\left(\Omega_{0}\right) \times L_{q}\left(\Omega_{1}\right) \longrightarrow L_{r, \infty}\left(\Omega_{2}\right)$ is compact. Therefore, $T\left(U_{L_{p}}, U_{L_{q}}\right)$ is relatively compact in $L_{r, \infty}\left(\Omega_{2}\right)$ and so it is also relatively compact in $\mathcal{M}\left(\mu_{2}\right)$. This proves (b) and completes the proof.

\section{Compactness of bilinear commutators of Calderón-Zygmund operators}

In this final section we work with the measure space $(\Omega, \mu)=\left(\mathbb{R}^{n}, d x\right)$. For this reason we drop the measure space in the notation for function spaces.

By a bilinear Calderón-Zygmund operator $T$ we mean a bounded bilinear operator $T: L_{p} \times L_{q} \longrightarrow L_{r}$ where $1<p, q<\infty, 1 / r=1 / p+1 / q$, such that there exits a kernel $K(x, y, z)$ defined away of the diagonal $x=y=z$ such that

and

$$
\begin{gathered}
|K(x, y, z)| \leq c \frac{1}{(|x-y|+|x-z|)^{2 n}}, \\
|\nabla K(x, y, z)| \leq c \frac{1}{(|x-y|+|x-z|)^{2 n+1}},
\end{gathered}
$$

$$
T(f, g)(x)=\int_{\mathbb{R}^{n}} \int_{\mathbb{R}^{n}} K(x, y, z) f(y) g(z) d y d z, \quad x \notin \operatorname{supp} f \cap \operatorname{supp} g
$$


where $f, g$ are bounded functions with compact support. See the paper by Grafakos and Torres [25] and the references given there.

Consider the following bilinear commutators

$$
\left\{\begin{array}{l}
{[T, b]_{1}(f, g)=T(b f, g)-b T(f, g),} \\
{[T, b]_{2}(f, g)=T(f, b g)-b T(f, g),} \\
{\left[\left[T, b_{1}\right]_{1}, b_{2}\right]_{2}(f, g)=\left[T, b_{1}\right]_{1}\left(f, b_{2} g\right)-b_{2}\left[T, b_{1}\right]_{1}(f, g) .}
\end{array}\right.
$$

where the functions $b, b_{1}, b_{2}$ belongs to $C M O$, the closure in $B M O$ of the space of $C^{\infty}$ functions with compact support.

Let $S$ be any of the bilinear commutators in (6.1). It has been shown by Lerner et al [36] and Pérez et al [41] that $S: L_{p} \times L_{q} \longrightarrow L_{r}$ is bounded for $1<p, q<\infty$ and $1 / r=1 / p+1 / q$, so $1 / 2<r<\infty$. Bényi and Torres [3, Theorem 1] have established compactness of $S$ provided $1 \leq r<\infty$. Next we use the interpolation results of the previous sections to show that $S: L_{p} \times L_{q} \longrightarrow L_{r}$ is also compact if $1 / 2<r<1$.

Theorem 6.1 Let $T$ be a bilinear Calderón-Zygmund operator, let $b, b_{1}, b_{2} \in C M O$ and let $S$ be any of the bilinear commutators defined in (6.1). If $1<p, q<\infty, 1 / 2<r<1$ and $1 / p+1 / q=1 / r$, then

$$
S: L_{p} \times L_{q} \longrightarrow L_{r} \quad \text { is compact. }
$$

Proof. Take $0<\varepsilon<\min (1-1 / 2 r, 1-1 / p, 1-1 / q)$ and put

$$
r_{1}=(1-\varepsilon) r, \quad p_{1}=(1-\varepsilon) p, \quad q_{1}=(1-\varepsilon) q .
$$

Then $1 / 2<r_{1}<r<1,1<p_{1}<p, 1<q_{1}<q$ and $1 / p_{1}+1 / q_{1}=1 / r_{1}$. Hence, according to [36, 41], $S: L_{p_{1}} \times L_{q_{1}} \longrightarrow L_{r_{1}}$ is bounded.

Choose $m \in \mathbb{N}$ such that $m r>1$ and write

$$
r_{0}=m r>1, \quad p_{0}=m p>p, \quad q_{0}=m q>q .
$$

Again $1 / p_{0}+1 / q_{0}=1 / r_{0}$ and, since $r_{0}>1$, it follows from [3, Theorem 1] that $S: L_{p_{0}} \times L_{q_{0}} \longrightarrow L_{r_{0}}$ is compact.

Next we show that $S$ may be uniquely extended to a bounded bilinear operator

$$
S:\left(L_{p_{0}}+L_{p_{1}}\right) \times\left(L_{q_{0}}+L_{q_{1}}\right) \longrightarrow L_{r_{0}}+L_{r_{1}} .
$$

Put $1 / s_{0}=1 / p_{0}+1 / q_{1}$ and $1 / s_{1}=1 / p_{1}+1 / q_{0}$. Then

$$
S: L_{p_{0}} \times L_{q_{1}} \longrightarrow L_{s_{0}} \text { and } S: L_{p_{1}} \times L_{q_{0}} \longrightarrow L_{s_{1}}
$$

are bounded. By our choices for parameters, we have that $1 / r_{0}<1 / s_{0}<1 / r_{1}$, so there is $0<\eta_{0}<1$ such that $1 / s_{0}=\left(1-\eta_{0}\right) / r_{0}+\eta_{0} / r_{1}$. Hence $L_{s_{0}} \hookrightarrow L_{r_{0}}+L_{r_{1}}$. On the other hand, since $1 / r_{0}<1 / s_{1}<1 / r_{1}$, there is $0<\eta_{1}<1$ such that $1 / s_{1}=\left(1-\eta_{1}\right) / r_{0}+\eta_{1} / r_{1}$. Whence $L_{s_{1}} \hookrightarrow L_{r_{0}}+L_{r_{1}}$. Consequently, the following restrictions are bounded

$$
\begin{aligned}
& S: L_{p_{0}} \times L_{q_{0}} \longrightarrow L_{r_{0}}+L_{r_{1}}, \\
& S: L_{p_{1}} \times L_{q_{1}} \longrightarrow L_{r_{0}}+L_{r_{1}}, \\
& S: L_{p_{0}} \times L_{q_{1}} \longrightarrow L_{r_{0}}+L_{r_{1}},
\end{aligned}
$$




$$
S: L_{p_{1}} \times L_{q_{0}} \longrightarrow L_{r_{0}}+L_{r_{1}}
$$

Now, proceeding as in Remark 4.10, we get that $S$ has a unique extension to a bounded bilinear operator

$$
S:\left(L_{p_{0}}+L_{p_{1}}\right) \times\left(L_{q_{0}}+L_{q_{1}}\right) \longrightarrow L_{r_{0}}+L_{r_{1}}
$$

as we claimed.

Next we choose $0<\theta<1$ such that $1 / r=(1-\theta) / r_{0}+\theta / r_{1}$. That is to say, satisfying that $1=(1-\theta) / m+$ $\theta /(1-\varepsilon)$. Then we also have that $1 / p=(1-\theta) / p_{0}+\theta / p_{1}$ and $1 / q=(1-\theta) / q_{0}+\theta / q_{1}$. Since

$$
S:\left(L_{p_{0}}, L_{p_{1}}\right) \times\left(L_{q_{0}}, L_{q_{1}}\right) \longrightarrow\left(L_{r_{0}}, L_{r_{1}}\right)
$$

with $S: L_{p_{0}} \times L_{q_{0}} \longrightarrow L_{r_{0}}$ compactly and $r_{0} \neq \infty$, applying Theorem 5.2 we conclude that

$$
S: L_{p} \times L_{q} \longrightarrow L_{r} \text { compactly. }
$$

Acknowledgements The authors have been supported in part by MTM2017-84058-P (AEI/FEDER, UE).

The authors would like to thank the referee for his/her remarks.

\section{References}

[1] C. Bennett and R. Sharpley, Interpolation of Operators, Academic Press, New York, 1988.

[2] Á. Bényi and T. Oh, Smoothing of commutators for a Hörmander class of bilinear pseudodifferential operators, J. Fourier Anal. Appl. 20 (2014) 282-300.

[3] Á. Bényi and R.H. Torres, Compact bilinear operators and commutators, Proc. Amer. Math. Soc. 141 (2013) 36093621.

[4] J. Bergh and J. Löfström, Interpolation Spaces. An introduction, Springer, Berlin, 1976.

[5] Y. Brudny̌̌ and N. Krugljak, Interpolation Functors and Interpolation Spaces, Vol. 1, North-Holland, Amsterdam, 1991.

[6] P.L. Butzer and H. Berens, Semi-Groups of Operators and Approximation, Springer, Berlin, 1967.

[7] A.P. Calderón, Intermediate spaces and interpolation, the complex method, Studia Math. 24 (1964) 113-190.

[8] A.P. Calderón and A. Zygmund, A note on the interpolation of linear operations, Studia Math. 12 (1951) $194-204$.

[9] F. Cobos, Interpolation theory and compactness, in: Function Spaces, Inequalities and Interpolation (Paseky 2009). Edited by J. Lukeš and L. Pick, Matfyzpress, Prague, 2009, pp. 31-75.

[10] F. Cobos, L.M. Fernández-Cabrera, A. Manzano and A. Martínez, Real interpolation and closed operator ideals, J. Math. Pures Appl. 83 (2004) 417-432.

[11] F. Cobos, L.M. Fernández-Cabrera and A. Martínez, Compact operators between K- and J-spaces, Studia Math. 166 (2005) 199-220.

[12] F. Cobos, L.M. Fernández-Cabrera and A. Martínez, Abstract $K$ and J spaces and measure of non-compactness, Math. Nachr. 280 (2007) 1698-1708.

[13] F. Cobos, T. Kühn and T. Schonbek, One-sided compactness results for Aronszajn-Gagliardo functors, J. Funct. Anal. 106 (1992) 274-313.

[14] F. Cobos and J. Peetre, Interpolation of compactness using Aronszajn-Gagliardo functors, Israel J. Math. 68 (1989) 220-240.

[15] F. Cobos and L.-E. Persson, Real interpolation of compact operators between quasi-Banach spaces, Math. Scand. 82 (1998) 138-160. 
[16] F. Cobos and A. Segurado, Description of logarithmic interpolation spaces by means of the J-functional and applications, J. Funct. Anal. 268 (2015) 2906-2945.

[17] M. Cwikel, Real and complex interpolation and extrapolation of compact operators, Duke Math. J. 65 (1992) $333-343$.

[18] M. Cwikel and J. Peetre, Abstract K and J spaces, J. Math. Pures Appl. 60 (1981) 1-50.

[19] D. E. Edmunds and B. Opic, Limiting variants of Krasnosel'skii’s compact interpolation theorem, J. Funct. Anal. 266 (2014) 3265-3285.

[20] W.D. Evans and B. Opic, Real interpolation with logarithmic functors and reiteration, Canad. J. Math. 52 (2000) 920 960.

[21] W.D. Evans, B. Opic and L. Pick, Real interpolation with logarithmic functors, J. Inequal. Appl. 7 (2002) $187-269$.

[22] D.L. Fernandez and E.B. da Silva, Interpolation of bilinear operators and compactness, Nonlinear Anal. 73 (2010) 526-537.

[23] L.M. Fernández-Cabrera and A. Martínez, On interpolation properties of compact bilinear operators, Math. Nachr. 290 (2017) 1663-1677.

[24] L.M. Fernández-Cabrera and A. Martínez, Real interpolation of compact bilinear operators, J. Fourier Anal. Appl. (2017). https://doi.org/10.1007/s00041-017-9561-7.

[25] L. Grafakos and R.H. Torres, Multilinear Calderón-Zygmund theory, Adv. Math. 165 (2002) 124-164.

[26] J. Gustavsson, A function parameter in connection with interpolation of Banach spaces, Math. Scand. 42 (1978) 289305.

[27] G. Hu, Compactness of the commutator of bilinear Fourier multiplier operator, Taiwanese J. Math. 18 (2014) 661-675.

[28] S. Janson, Minimal and maximal methods of interpolation, J. Funct. Anal. 44 (1981) 50-73.

[29] S. Janson, On interpolation of multi-linear operators, in Function Spaces and Applications. Edited by M. Cwikel, J. Peetre, Y. Sagher and H. Wallin, Springer Lect. Notes Math. 1302, Berlin, 1988, pp.290-302.

[30] G.E. Karadzhov, The interpolation method of "means" for quasinormed spaces, Doklady Acad. Nauk SSSR 209 (1973) 33-36.

[31] H. König, On the tensor stability of s-number ideals, Math. Ann. 269 (1984) 77-93.

[32] H. König, Eigenvalue Distribution of Compact Operators, Birkhäuser, Basel, 1986.

[33] G. Köthe, Topological Vector Spaces I, Springer, Berlin, 1969.

[34] M.A. Krasnosel'skii, On a theorem of M. Riesz, Soviet Math. Dokl. 1 (1960) 229-231.

[35] M.A. Krasnosel'skii, P.P. Zabreiko, E.I. Pustylnik and P.E. Sbolevskii Integral operators in spaces of summable functions, Noordhoff, Leyden, 1976.

[36] A.K. Lerner, S. Ombrosi, C. Pérez, R.H. Torres and R. Trujillo-González, New maximal functions and multiple weights for the multilinear Calderón-Zygmund theory, Adv. Math. 220 (2009) 1222-1264.

[37] J.-L. Lions and J. Peetre, Sur une classe d'espaces d'interpolation, Inst. Hautes Études Sci. Publ. Math. 19 (1964) 5-68.

[38] P. Nilsson, Reiteration theorems for real interpolation and approximation spaces, Ann. Mat. Pura Appl. 132 (1982) 291-330.

[39] P. Nilsson, Interpolation of Calderón and Ovčnnikov pairs, Ann. Mat. Pura Appl. 134 (1983) 201-232.

[40] J. Peetre, A theory of interpolation of normed spaces, Lecture Notes, Brasilia, 1978.

[41] C. Pérez, G. Pradolini, R.H. Torres and R. Trujillo-González, End-point estimates for iterated commutators of multilinear singular integrals, Bull. London Math. Soc. 46 (2014) 26-42.

[42] A. Persson, Compact linear mappings between interpolation spaces, Ark. Mat. 5 (1964) 215-219.

[43] L.-E. Persson, Interpolation with a parameter function, Math. Scand. 59 (1986) 199-222.

[44] H. Triebel, Interpolation Theory, Function Spaces, Differential Operators, North-Holland, Amsterdam, 1978.

[45] H. Triebel, Theory of Function Spaces II, Birkhäuser, Basel, 1992. 